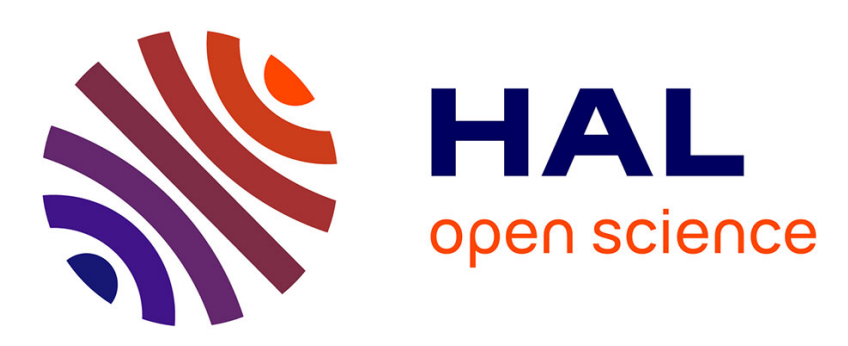

\title{
On the mechanics of the global bending collapse of circular tubes under dynamic axial load - Dynamic buckling transition
}

D. Karagiozova, Norman Jones

\section{- To cite this version:}

D. Karagiozova, Norman Jones. On the mechanics of the global bending collapse of circular tubes under dynamic axial load - Dynamic buckling transition. International Journal of Impact Engineering, 2008, 35 (5), pp.397. 10.1016/j.ijimpeng.2007.04.002 . hal-00499101

\section{HAL Id: hal-00499101 \\ https://hal.science/hal-00499101}

Submitted on 9 Jul 2010

HAL is a multi-disciplinary open access archive for the deposit and dissemination of scientific research documents, whether they are published or not. The documents may come from teaching and research institutions in France or abroad, or from public or private research centers.
L'archive ouverte pluridisciplinaire HAL, est destinée au dépôt et à la diffusion de documents scientifiques de niveau recherche, publiés ou non, émanant des établissements d'enseignement et de recherche français ou étrangers, des laboratoires publics ou privés. 


\section{Author's Accepted Manuscript}

On the mechanics of the global bending collapse of circular tubes under dynamic axial load - Dynamic buckling transition

D. Karagiozova, Norman Jones

PII: S0734-743X(07)00062-0

DOI: doi:10.1016/j.ijimpeng.2007.04.002

Reference: IE 1478

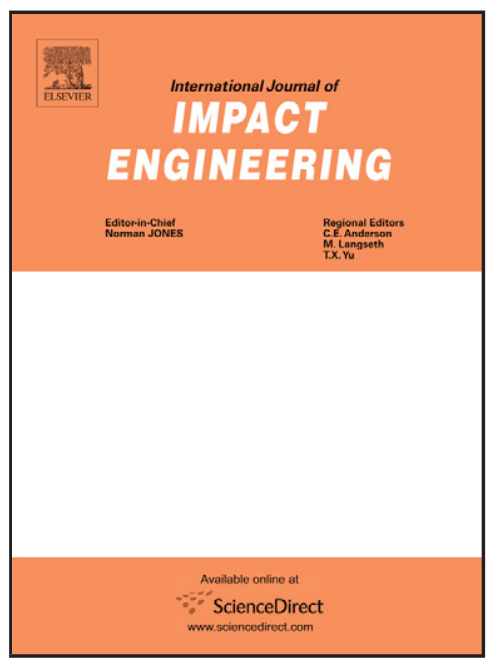

www.elsevier.com/locate/ijimpeng

To appear in: International Journal of Impact

Accepted date: $\quad 10$ April 2007

Cite this article as: D. Karagiozova and Norman Jones, On the mechanics of the global bending collapse of circular tubes under dynamic axial load - Dynamic buckling transition, International Journal of Impact (2007), doi:10.1016/j.ijimpeng.2007.04.002

This is a PDF file of an unedited manuscript that has been accepted for publication. As a service to our customers we are providing this early version of the manuscript. The manuscript will undergo copyediting, typesetting, and review of the resulting galley proof before it is published in its final citable form. Please note that during the production process errors may be discovered which could affect the content, and all legal disclaimers that apply to the journal pertain. 


\title{
On the mechanics of the global bending collapse of circular tubes under dynamic axial load - Dynamic buckling transition
}

\author{
D. Karagiozova* \\ Institute of Mechanics, Bulgarian Academy of Sciences \\ Acad. G. Bonchev Street, Block 4, Sofia 1113, Bulgaria \\ Norman Jones \\ The University of Liverpool, Department of Engineering \\ Brownlow Hill, Liverpool L69 3GH, UK
}

\begin{abstract}
The common features of the deformation of long circular tubes made from different aluminium alloys and subjected to various axial impact loadings are established and used to develop simplified structural models for global bending collapse and progressive buckling. A two-phase deformation model for axially loaded structural elements is proposed, which retains the characteristic features of the initial compression and subsequent bending/buckling phases. It is shown that the velocity histories in the two principal collapse modes of long tubes play an important role for the formation of the particular deformation pattern. The simplified structural models are verified with some numerical finite-element results and their behaviour is shown to be adequate.

A parametric analysis of the models is further performed in order to divulge the major factors, which influence the dynamic buckling transition. The analysis reveals that there is a specific impact velocity associated with the particular geometric and material properties of a circular tube, which causes a counter-intuitive response. An empirical criterion for the lower and upper bounds to the critical impact velocity for a buckling transition is proposed. Further, the upper bound to the impact velocity is used to formulate a criterion for the dynamic buckling transition.
\end{abstract}

Key words: Global bending, Progressive buckling, Axial impact, Circular tubes, Buckling transition, Transition criterion

\footnotetext{
${ }^{*}$ Corresponding author: D. Karagiozova

E-mail: d.karagiozova@imbm.bas.bg

Fax: Fax: +359 28707498
} 


\section{Nomenclature}

A

$D, h, L$

$E, E_{h}$

$F(t)$

G

$G_{1}$

l

$m$

$m_{1}$

$M$

$M_{p}$

$t^{*}$

$t_{\text {glob }}^{*}, t_{\text {progr }}^{*}$

$u, u_{C}$

$T_{p, f}$

$T_{0}$

$V_{s f}$

$V_{\text {str }}$

$V_{0}$

$V_{0, c i}$

$V_{0, c r}$

$V_{0, t}$

$V_{1, \text { glob }}, V_{1, \text { progr }}$

$w, W$

$x, \theta, z$

$\delta$

$\Delta$

$\lambda$

$\varphi(t), \dot{\varphi}(t)$

$\varphi_{0}$

$\phi_{1}, \phi_{2}$

$\Phi(t), \dot{\Phi}(t)$

$\psi(t), \dot{\psi}(t)$

$\psi_{0}$ cross-sectional area of a tube

outer diameter, thickness and length of a circular tube, respectively

Young's modulus and strain hardening modulus, respectively

axial force at the proximal end of a shell

striking mass

$G / \pi D$, striking mass per unit circumferential length

length of a single axisymmetric fold, $2(\pi R h / \sqrt{3})^{1 / 2}$, [2]

$\pi D h \rho$, mass per unit length of a shell, Fig. 6(a)

$\rho h l$, mass per unit circumferential length of a single fold, Fig. 6(b)

mass of a shell

fully plastic bending moment per unit circumferential length, $\sigma_{0} h^{2} / 4$

duration of the compression phase of a tube

duration of the compression phases of global and progressive buckling modes,

respectively

vertical displacements at the proximal end of a tube (Figs. 6(a), 6(b)) and at the distal end of the first axisymmetric fold (Fig. 6(b)), respectively

plastic energy absorbed by a single axisymmetric fold

initial impact energy

axial velocity related to the energy dissipation by a single axisymmetric fold, Eq. (36)

velocity of the striker, Eq.(3)

impact velocity

impact velocity causing a counter-intuitive response of a tube

critical impact velocity causing entirely progressive buckling response

impact velocity at the initiation of buckling transition

vertical velocities of the models associated with global bending and progressive

buckling, respectively, Fig. 6(a) and 6(b)

transverse and lateral displacements for progressive buckling and global bending modes, respectively

axial, circumferential and through-the-thickness coordinates of a shell, respectively

overall reduction of the length of a single axisymmetric fold during the compression

phase of deformation, Fig. 6(b)

overall reduction of the shell length during the compression phase of deformation,

Fig. 6(a)

$E_{h} / E$

angles and angular velocities associated with the compression phase of a global

bending mode, Fig. 6(a)

initial imperfections for the global bending (compression phase, Fig. 6(a))

characteristic angles for the bending phase of the global bending mode

angles and angular velocities for a global bending mode during the large deformation phase, Fig. 7(a)

angles and angular velocities associated with the compression phase of a progressive buckling mode, Fig. 6(b)

initial imperfections for the progressive buckling mode (compression phase, Fig. 6(b)) 


$\begin{array}{ll}\Psi(t), \dot{\Psi}(t) & \begin{array}{l}\text { angle and angular velocity for a progressive buckling mode during the large } \\ \text { deformation phase, Fig. } 7(\mathrm{~b})\end{array} \\ \sigma_{0} & \text { yield stress } \\ \sigma^{0}(t) & \text { flow stress }\end{array}$

\section{Introduction}

In the optimum design of tubes with various cross-sections for energy absorption [1], it is usually assumed that the particular geometry will promote a progressive buckling mechanism [2,3], which is maintained throughout the response. It is also anticipated that an introduction of a mechanical dent (trigger) is sufficient to control formation of the first fold when a shell-like structure is loaded quasistatically.

The initiation of the desired buckling mode becomes more complicated when long shell-like structures are used to absorb the impact energy. Such slender structures can exhibit additional buckling modes leading to poor energy absorption. In particular, buckling modes similar to the Euler buckling mode characteristic for statically loaded columns or higher dynamic buckling modes can develop in long tubes (global bending) as shown experimentally by Andrews et al. [4], Abramowicz and Jones [5] and other authors [6-8]. The particular mode of collapse depends on the geometry, boundary conditions and material of the structure. Recently, extensive numerical work has provided some insight into the dynamic buckling transition from global bending collapse to progressive buckling $[7,9,10]$.

The threshold conditions between a quasi-static progressive buckling and global bending of circular tubes depend on the ratios $D / h$ and $L / D$, as first reported in [4] for aluminium tubes and recently in [11] for aluminium alloy tubes. It was shown experimentally that a static critical tube length for a buckling transition exists for a particular ratio, $D / h$, so that tubes shorter than this critical length collapse progressively, while longer tubes develop a global bending mode.

Similar observations were reported by Abramowicz and Jones [5] who studied the response of long circular and square mild steel tubes and by Jensen et al. [7] for dynamically loaded square aluminium alloy extrusions. The experimental $[5,8]$ and numerical $[7,9,10]$ results for circular and square tubes, subjected to axial impacts with initial velocities up to $20 \mathrm{~m} / \mathrm{s}$, show that the critical length for the buckling transition has a tendency to increase when increasing the impact velocity, although, for some loading parameters, a counter-intuitive response is observed and no critical buckling length can be defined.

Due to the complexity of the dynamic buckling transition, quasi-static methods have been used in the existing analytical models to establish criteria for the transition between progressive and global buckling, as reported by Abramowicz and Jones [5] and Mahmood and Paluszny [12]. Recently, a theoretical analysis of the influence of various factors on the dynamic buckling of long tubes was reported, which explains some of the phenomena related to the dynamic buckling transition [10]. 
However, the analysis focused on the global bending collapse and the conditions under which progressive buckling can develop were not considered.

The purpose of the present study is to analyse the development of the two above mentioned buckling modes in relatively long circular tubes in order to obtain insight into the transition from global bending to progressive buckling collapse for dynamic axial loads.

Simplified structural models of the two buckling modes are proposed and used to explore the influence of the material properties and loading parameters on the dynamic buckling mechanism during the initial compression and subsequent post-buckling response. An empirical criterion for the lower and upper bounds to the critical impact velocity for a transition between the two buckling modes is proposed. Further, the upper bound is used as a criterion for the critical impact velocity causing an entirely progressive buckling collapse of an axially loaded circular tube.

\section{Numerical model}

The experimental observations on the buckling transition $[4,5,7,8]$ can be used to obtain empirical estimates of the critical length at the transition conditions for some particular cases using curve-fitting techniques. However, they cannot predict the influence of the various parameters on the dynamic buckling transition. On the other hand, a numerical simulation of the behaviour of long tubes subjected to axial impact is a suitable complementary examination of the problem, which allows for a more detailed analysis during deformation. For that reason, it is useful to perform numerical simulations with various parameters in order to provide an insight into the mechanics of the buckling transition. The simulations reported here were carried out using the FE code ABAQUS/Explicit and they serve as a reference for the subsequent simplified semi-analytical solution. It is assumed that a tube freely standing on a rigid surface is struck by a rigid mass. Shell elements RS4 (approximately $3.9 \mathrm{~mm} \times 4 \mathrm{~mm}$ ) were used to model the tubes. The mesh sensitivity analysis showed that the further mesh refinement affected only slightly the critical impact velocity for buckling transition when all other model parameters were kept constant. The load is applied as a point mass attached to the nodes of a rigid body which had an initial velocity, $V_{0}$. The contact between the shell and striker and between the distal end of the shell and rigid surface was defined using the 'surface interaction' concept together with a friction coefficient of 0.25 at both ends. Any self-contacts of the inner and the outer surfaces of the shell were assumed frictionless.

In order to trigger asymmetric buckling patterns, initial imperfections corresponding to the first two elastic buckling modes of a shell were introduced with a maximum amplitude of $0.0005 \mathrm{~L}$. The selected magnitude is consistent with the standard manufacturing tolerance for aluminium extrusions, which prescribes a maximum deviation of $0.001 \mathrm{~L}$ with respect to the longitudinal axis of a circular tube [13]. The numerical model was verified using the experimental data reported in [9] and a typical example is shown in Fig. 1 (the actual stress-strain curve for an aluminium alloy is presented in Fig. 
A2.2). It should be emphasised that no additional initial imperfections were introduced in the model to trigger directly axisymmetric progressive buckling and the numerically obtained transition is caused only by the variation of the loading parameters.

The material characteristics used in the present study (see Table 1) were selected to understand the influence of the material properties on the buckling transition. Mat1 and Mat4 have equal but relatively low ratios of $E_{h} / \sigma_{0}$, while Mat3 has a low value of the yield stress $\sigma_{0}$, but a much larger ratio $E_{h} / \sigma_{0}$.

\section{Transition from progressive buckling to global bending collapse for dynamic loads}

The experiments reported in the literature on the dynamic buckling of relatively long tubes under axial impacts with various masses and initial velocities $[5,6,8]$ and the numerical simulations on the same problem $[7,9,10]$ show that several buckling modes exist, which depend on the material properties, loading parameters and the geometry of the shells. In general, three patterns of buckling are observed. Global bending (Figs. 2(a), 2(c), 2(e)) has the characteristics of a buckled long column where plastic 'bending hinges' develop due to the ovalisation of the shell cross-section. The progressive buckling mode (Figs. 2(b), 2(d), 2(f), 2(g)) is characterised by folds, which develop sequentially. And finally, the mixed buckling mode (Fig. 1(h)) is a combination of progressively developed folds and global bending. The influence of the material properties on the position of the 'bending hinges' is shown in Fig. 2(a) and 2(c), while the stabilisation effect of the higher impact velocity on the buckling of a tube with a constant length of $L \approx 9 D$ is demonstrated in Fig 2(e,f). A transition from progressive buckling mode to a mixed buckling mode when increasing the tube length is shown in Fig. 2(g,h).A low velocity impact can cause a buckling mode with the characteristics of the Euler buckling mode as shown in Fig. 2(c), while, in general, a higher impact velocity increases the critical length for the transition from the column to a shell buckling mode $[5,9,10]$ causing the development of column buckling modes higher than the static Euler buckling mode (Figs. 2(a), 2(e)). The shells in Figs. 2(a) 2(f) have an outer diameter $D=50.8 \mathrm{~mm}$ and thickness $h=2 \mathrm{~mm}$, while the corresponding characteristics of the shells in Figs. $2(\mathrm{~g})$ and $2(\mathrm{~h})$ are $D=50.2 \mathrm{~mm}$ and thickness $h=2.19 \mathrm{~mm}$. The material characteristics of these tubes are listed in Table 1.

Despite the variety of final shapes, a common feature of long tubes subjected to axial impact is the presence of two phases of deformation - compression and bending, which always occur regardless of the buckling pattern. Fig. 3 shows numerically obtained displacement-time histories at some particular points along a tube marked in Fig. 4(a). It is evident that lateral displacements associated with local folds, $w$, (top and bottom of a shell, points ' $t$ ' and ' $b$ ' are at the tips of the corresponding folds) and with global bending, $W$ (m, at a bending hinge), start to develop simultaneously during the compression phase for $t<0.001 \mathrm{~s}$, although the corresponding magnitudes remain small. The dominant buckling mode starts to grow more rapidly during the bending phase, which, in some cases, 
leads to the corresponding final buckling mode when global bending or progressive buckling develops. Sometimes, the initially formed progressive buckling mode can switch to a global bending after the formation of one or more folds, which forms the mixed buckling mode.

It is also observed from the numerical simulations that the duration of the compression phase of shells with $D / h=$ const and made from the same material depends on the length of the tube and the impact velocity. This dependence is illustrated in Fig. 5. The compression phase in longer tubes has a longer duration for both modes (global bending in Fig. 5(a) and progressive buckling in Fig. 5(b)). An increase of the impact velocity causes a more rapid buckling response as shown in Fig. 5(c) for two progressive buckling cases.

The response of a long tube to an axial impact implies that the initial conditions at the onset of the bucking/bending phase are key factors for the development of the particular collapse mode during the post-collapse phase. The observed features of the tube response suggest that, as a reasonable approximation, the compression and bending phases can be de-coupled and analysed sequentially. Small lateral displacements and considerable compression develop during the first phase, while large transverse or lateral displacements and, therefore, large rotations develop during the second phase without any significant compression. This behaviour allows certain simplifications to be introduced in the possible models while preserving the essential features of the deformation process as a whole.

\section{Simple models for the buckling modes}

\subsection{Two-phase approach for deformation of axially loaded elastic-plastic structures}

The axially loaded shells can be classified as 'Type II' structures, which are velocity and mass sensitive [14-17]. The two-phase approach for an analysis of the deformation of these structures was proposed by Tam and Calladine [14] and is used here to examine the response of relatively long circular tubes subjected to axial impact loadings by a heavy mass travelling with an initial velocity. The basic assumption used in this approach is that the initial phase of compression can be separated from the subsequent bending phase. The compression phase develops under continuous loading when small lateral/transverse displacements build up within a sustained axial plastic flow, so that no elastic unloading due to bending occurs. The duration of the compression phase depends on how long the lateral/transverse inertia can support the unbuckled shape $[7,8,17,18]$, so that the collapse mode that occurs in a long tube under axial impact depends not only on the geometry of the tube, as in the static case [4], but also on the loading parameters. In addition, the transverse displacements and velocities at the end of the compression phase also depend on the material properties of the shell.

By way of contrast, large bending deformations develop during the second phase and form "plastic hinges". Depending on the loading configuration, different initial conditions occur in a shell at the onset of the second phase of deformation, which can be either progressive buckling or global 
bending. Thus, it is essential to find relations between the various tube characteristics and loading parameters at the buckling transition.

\subsection{Initiation of buckling}

Circular tubes with characteristic ratios $L / D$ between 7 and 20 (Fig. 2 and Fig 5) analysed in the present study responded predominantly with the second overall buckling mode of a column unless conditions for the development of progressive buckling were satisfied. This buckling characteristic is also evident from the experimental results in Fig. 1(c) and from the final deformation shapes of circular tubes shown in [8]. Therefore, a column mode with these characteristics was selected for the analysis of the global bending mode (Fig. 4(a)). An axisymmetric fold (Fig. 4(b)) initiates for the shell buckling mode near to the proximal or distal ends of a shell in many axial impact cases during the development of progressive buckling. Therefore, the deformed shapes in Fig. 4 are anticipated as the basic characteristic shapes, which can occur in a long tube under an axial impact.

Two separate structural models for the initiation of a global bending (Fig 6(a)) and for the initiation of a local fold (Fig 6(b)) are proposed in order to obtain the conditions for the development of the two principal buckling modes, which depend on the material and loading parameters and the length of a shell.

\subsubsection{Global bending}

The model in Fig. 6(a) represents the second axial buckling mode of a long circular shell and consists of three links, each of length $L / 3$ with a distributed mass $m=M / L$. The links are axially compressible by an amount $\Delta / 3$, but do not deform due to the bending moments. The initial imperfections of the model have the expected buckling mode shape, so that it is assumed that $W_{0}=\varphi_{0} L / 3$ with opposite signs at the ends of the first and second links. The model deforms due to an axial impact by a mass $G$ with an initial velocity $V_{0}$ and is assumed to produce a continuous loading across the shell cross-section. According to Ref. [14], the velocity of the proximal end of the model in Fig. 6 (a) can be expressed as a sum of the velocity related to the rotation of the links, in the present case, $V_{1, g l o b}$, and the velocity due to compression, $\dot{\Delta}$

$$
V=\dot{u}=V_{1, \text { glob }}+\dot{\Delta},
$$

where $u$ is the displacement at the proximal end of this model and can be expressed as a function of the rotational angles $\varphi_{1}, \varphi_{2}$, initial imperfections $\varphi_{01}, \varphi_{02}$ and the geometric and material characteristics of a shell. It is shown in [10] that for the special case of $\varphi_{1}=\varphi_{2}=\varphi, \varphi_{01}=\varphi_{02}=\varphi_{0}$ and a small rotational angle $\varphi \approx 3 W / L$, the velocity due to the rotation of the links is 


$$
V_{1, g l o b}(t)=\frac{9 \gamma W_{0}^{2}}{L} \sinh (2 \gamma t)
$$

where $\gamma=\frac{3}{L} \sqrt{\frac{6 \sigma_{0}}{\rho}} \sqrt{1-\frac{30 E_{h} R^{2}}{\sigma_{0} L^{2}(1+\sqrt{\lambda})^{2}}}$ and $E_{h}$ is the strain hardening modulus.

It is assumed that there is no separation of the striker during impact and from the equality $G \dot{V}_{\text {str }}=-A \sigma_{0}$ the velocity of the striker is obtained

$$
V_{s t r}(t)=V_{0}-\frac{A \sigma_{0}}{G} t
$$

where $A=\pi D h$ is the cross-sectional area of the shell.

The two-phase approach [14] for structures sensitive to the impact velocity and inertia effects states that the compression phase is defined by the inequality

$$
V_{1, g l o b} \leq V_{\text {str }} \quad \text { for } \quad 0 \leq t \leq t_{\text {glob }}^{*}
$$

where $t_{\text {glob }}^{*}$ marks the end of this phase when $V_{1, \text { glob }}\left(t_{\text {glob }}^{*}\right)=V_{\text {str }}\left(t_{\text {glob }}^{*}\right)$. Equation (2) is not valid for $t>t_{\text {glob }}^{*}$ since the subsequent phase of bending involves large rotational angles and large bending deformations causing elastic unloading and eventually plastic reloading across the thickness.

\subsubsection{Progressive buckling}

A model for the initiation of a local fold is shown in Fig. 6(b). It is assumed, as for the previous model, that deformation develops under a continuous loading, buckling occurs within a sustained axial plastic flow and the rotations of the links remain small. This model represents an axisymmetric fold with length $l=2 \tilde{l} \quad\left(\tilde{l}=(\pi h R / \sqrt{3})^{1 / 2}[2]\right)$ of a circular shell with length $L$ and considers the initial deformation of a strip having a unit circumferential length. The equation of motion for the model is

$$
\frac{\rho h l^{3}}{24} \ddot{\psi}+M_{A}+\frac{M_{B}}{2}+M_{r}+\frac{N_{x} l}{2} \psi=0
$$

when taking into account that $M_{A}=M_{C}$ in Fig. 6(b). In Eq.(5), $N_{x}$ is the axial force in the links, $M_{A}, M_{B}$ are the bending moments at $\mathrm{A}$ and $\mathrm{B}$ and $M_{r}$ is the bending moment resulting from the circumferential forces in an actual shell.

Let us consider a circular shell struck by a large mass travelling with an initial velocity $V_{0}$ which causes the development of an axisymmetric fold near to the proximal end. A plane stress field $\sigma_{x} \neq 0, \sigma_{\theta} \neq 0$ is assumed and the stresses are defined by the Levi-Mises equations with an isotropic hardening

$$
\sigma_{x}=\frac{2 \sigma_{e}}{3 \dot{\varepsilon}_{e}}\left(2 \dot{\varepsilon}_{x}+\dot{\varepsilon}_{\theta}\right) \text { and } \sigma_{\theta}=\frac{2 \sigma_{e}}{3 \dot{\varepsilon}_{e}}\left(2 \dot{\varepsilon}_{\theta}+\dot{\varepsilon}_{x}\right)
$$


where the equivalent stress is $\sigma_{e}=\sigma_{0}+E_{h} \varepsilon_{e}$. The associated total strain rates according to the LoveKirchhoff assumptions are [19]

$$
\dot{\varepsilon}_{x}=-\frac{V_{0}}{L}-z \frac{\partial^{2} \dot{w}}{\partial x^{2}}, \quad \dot{\varepsilon}_{\theta}=\left(1-\frac{z}{R}\right)\left(\frac{V_{0}}{2 L}+\frac{\dot{w}}{R}\right) \quad \text { and } \quad \dot{\varepsilon}_{z}=-\dot{\varepsilon}_{x}-\dot{\varepsilon}_{\theta} .
$$

For small transverse displacements $w=\psi \tilde{l}$.

Under the assumption of a continuous axial compression, the stress field in the shell becomes

$$
\begin{aligned}
& \sigma_{x}=\frac{2 \sigma_{e}}{3 \dot{\varepsilon}_{e}}\left[-\frac{3 V_{0}}{2 L}-z\left(\frac{V_{0}}{2 R L}+\frac{\dot{\psi}}{\widetilde{l}}\right)\right], \quad \sigma_{\theta}=\frac{2 \sigma_{e}}{3 \dot{\varepsilon}_{e}}\left[-z\left(\frac{V_{0}}{R L}+\frac{\dot{\psi}}{\widetilde{l}}\right)\right] \\
& \dot{\varepsilon}_{e} \cong \frac{V_{0}}{L}\left[1+2 \frac{z L}{V_{0}} \frac{\dot{\psi}}{\widetilde{l}}\right]^{1 / 2}, z \in[0, h]
\end{aligned}
$$

at position $\mathrm{A}$,

$$
\begin{aligned}
& \sigma_{x}=\frac{2 \sigma_{e}}{3 \dot{\varepsilon}_{e}}\left[-\frac{3 V_{0}}{2 L}+\frac{\tilde{l}}{R} \dot{\psi}-z\left\{\frac{V_{0}}{2 R L}-\dot{\psi}\left(\frac{2}{\widetilde{l}}-\frac{\tilde{l}^{2}}{R}\right)\right\},\right. \\
& \sigma_{\theta}=\frac{2 \sigma_{e}}{3 \dot{\varepsilon}_{e}}\left[\frac{2 \tilde{l}}{R} \dot{\psi}-z\left\{\frac{V_{0}}{2 R^{2}}-\dot{\psi}\left(\frac{2}{\widetilde{l}}-\frac{\tilde{l}}{R^{2}}\right)\right\},\right. \\
& \dot{\varepsilon}_{e} \cong \frac{V_{0}}{L}\left[1-4 \frac{z L}{V_{0}}\left(\frac{1}{3 R^{2}}+\frac{1}{\widetilde{l}^{2}}\right) \dot{\psi} \tilde{l}\right]^{1 / 2}, \quad z \in[-h, 0]
\end{aligned}
$$

at position $\mathrm{B}$, where $\sigma_{e}=\sigma_{0}+E_{h} \varepsilon_{e}, \varepsilon_{e}=\int_{0}^{t} \dot{\varepsilon}_{e} d t$. The links $\mathrm{AB}$ and $\mathrm{BC}$ are compressible but rigid in bending, so it is anticipated that the stresses and the equivalent strain rate for these links are equal to the corresponding values at their mid-length

$$
\begin{aligned}
& \sigma_{x}=\frac{2 \sigma_{e}}{3 \dot{\varepsilon}_{e}}\left[-\frac{3 V_{0}}{2 L}+\frac{\tilde{l}}{2 R} \dot{\psi}-z\left(\frac{V_{0}}{2 R L}+\frac{\dot{\psi} \tilde{l}}{R}\right)\right], \sigma_{\theta}=\frac{2 \sigma_{e}}{3 \dot{\varepsilon}_{e}}\left[\frac{\tilde{l}}{R} \dot{\psi}-z\left(\frac{V_{0}}{2 R L}+\frac{\tilde{l} \dot{\psi}}{R^{2}}\right)\right] \\
& \dot{\varepsilon}_{e} \cong \frac{V_{0}}{L}\left[1-\frac{z L}{V_{0}} \frac{\tilde{l}}{3 R^{2}} \dot{\psi}\right]^{1 / 2}, z \in[-h / 2, h / 2] .
\end{aligned}
$$

The forces and moments associated with the model in Fig. 6(b) are the integrals of the corresponding stresses according to Eqs.(8-10). After neglecting the higher order terms, they are obtained at several positions as

$$
\begin{aligned}
& M_{x}=-\int_{0}^{h} \sigma_{x}(1+z / R) z d z \cong \sigma^{0} \frac{[2+(b h-2) \sqrt{1+b h}]}{3 b^{2}}+f_{x}\left(E_{h}, \varepsilon_{e}\right), \\
& N_{\theta}=\int_{0}^{h} \sigma_{\theta} d z \cong-\frac{4 \sigma^{0}}{3}\left(\frac{1}{R}+\frac{\dot{\psi}}{\tilde{l}}\right) \frac{[2+(b h-2) \sqrt{1+b h}]}{3 b^{2}}+f_{\theta}\left(E_{h}, \varepsilon_{e}\right),
\end{aligned}
$$

at position A, where $b=2 \frac{L}{V_{0}} \frac{\dot{\psi}}{\widetilde{l}}$. 


$$
\begin{aligned}
& M_{x}=-\int_{-h}^{0} \sigma_{x}(1+z / R) z d z \cong 2 \sigma^{0}\left(1+\frac{2 \tilde{l}}{3 R} \dot{\psi}\right) \frac{[2-(2-b h) \sqrt{1+b h}]}{3 b^{2}}+f_{x}\left(E_{h}, \varepsilon_{e}\right), \\
& N_{\theta}=\int_{-h}^{0} \sigma_{\theta} d z \cong-\sigma^{0} \frac{8 \tilde{l} \sigma_{e}}{3 R L b} \dot{\psi}[1-\sqrt{1+b h}]+f_{\theta}\left(E_{h}, \varepsilon_{e}\right),
\end{aligned}
$$

at position B with $b=4 \frac{L}{V_{0}}\left(\frac{1}{3 R^{2}}+\frac{1}{\widetilde{l}^{2}}\right) \dot{\psi}$ and

$$
N_{x}=\int_{-h / 2}^{h / 2} \sigma_{x} d z \cong-\sigma^{0} h+\frac{\sigma^{0} L h \tilde{l}}{3 R V_{0}} \dot{\psi}, \quad N_{\theta}=\int_{-h / 2}^{h / 2} \sigma_{\theta} d z \cong \frac{2 \sigma^{0} \tilde{l} h L}{3 R V_{0}} \dot{\psi}
$$

at the middle of the compressible link $\mathrm{AB}, \sigma^{0}=\sigma_{0}+E_{h} V_{0} t / L$. The functions $f_{x}\left(E_{h}, \varepsilon_{e}\right)$ and $f_{\theta}\left(E_{h}, \varepsilon_{e}\right)$ in Eqs.(11), (12) cannot be presented in a closed form but they can be obtained approximately in a discrete form. However, $f_{x}\left(E_{h}, \varepsilon_{e}\right)$ and $f_{\theta}\left(E_{h}, \varepsilon_{e}\right)$ can be neglected for small material strain hardening.

Equation (5) is solved numerically using the Runge-Kutta method (multiple orders) within intervals $\Delta t(t=N \Delta t)$ when using the initial conditions

$$
\psi(0)=\psi_{0} \text { and } \dot{\psi}(0)=0 \text {. }
$$

The displacement of the proximal end of the model in Fig. $6(\mathrm{~b})$ is

$$
u=\left(\psi^{2}-\psi_{0}^{2}\right) l / 2+\delta+u_{C},
$$

where $\delta$ is the overall reduction in the length of a single fold due to axial compression and $u_{C}$ is the vertical displacement of point $\mathrm{C}$ due to the uniform compression without any buckling of the shell between $\mathrm{C}$ and the distal end. A shell can deform potentially with $n \approx L / l$ folds. The duration of the compression phase for progressive buckling is based on the relative vertical velocities of points $\mathrm{C}$ and A, where point A moves at a greater vertical speed due to the formation of a local fold. The velocity of the proximal end of the model in Fig. 6(b) is

$$
V_{\text {Model }}(t)=\widetilde{V}_{1, \text { progr }}(t)+\dot{\delta}+V_{C}(t),
$$

where $V_{C}(0)=V_{0}(n-1) / n$ and when assuming that the cross-section at $\mathrm{C}$ continues to move downwards at a speed proportional to the speed of the striker during the compression phase. The velocity due to the rotation of the links is

$$
\widetilde{V}_{1, p r o g r}(t)=2 \psi \dot{\psi} \tilde{l}
$$

and

$$
V_{C}(t)=V_{s t r}(t)(n-1) / n
$$

The latter indicates that the part of a shell below the cross-section at point $\mathrm{C}$ undergoes continuous compression without any buckling. During the compression phase for the progressive buckling mode, the following condition is satisfied 


$$
\widetilde{V}_{1, p r o g r} \leq V_{s t r}(t)-V_{C}(t) \quad \text { for } \quad 0 \leq t \leq t_{\text {progr }}^{*}
$$

Thus, the vertical velocity at the proximal end of the model, which is characteristic for the compression phase of the progressive buckling mode, is

$$
V_{1, \text { progr }}(t)=\widetilde{V}_{1, \text { progr }}(t)+V_{C}(t)
$$

and at the end of this phase

$$
V_{1, \text { progr }}\left(t_{\text {progr }}^{*}\right)=V_{\text {str }}\left(t_{\text {progr }}^{*}\right) \text {. }
$$

\subsection{Post-buckling response}

Two separate models for the post-buckling response of an actual shell, which describe the development of a global collapse (Fig 7(a)) and a local fold (Fig 7(b)), are proposed in order to analyse the influence of the loading parameters and shell parameters on the dynamic buckling transition. The links are rigid and incompressible in both models, and the energy can dissipate only due to rotation at the hinges. The rotational angles $\varphi\left(t^{*}\right)$ and $\psi\left(t^{*}\right)$, and angular velocities $\dot{\varphi}\left(t^{*}\right), \dot{\psi}\left(t^{*}\right)$, which are obtained at the end of the compression phase, are used as initial conditions for the corresponding post-buckling phase of global bending or progressive buckling.

\subsubsection{Global bending}

In a manner similar to the initial compression phase, the bending phase is analysed for the second column bending mode Fig. 7(a) with $\phi_{1}=\phi_{2}=\phi$. The total kinetic energy of this model in axial and transverse directions is

$$
T=\frac{L^{2} \dot{\Phi}^{2}}{216\left(4-\sin ^{2} \Phi\right)}\left\{12 G \sin ^{2}(\phi+\Phi)+M\left[1+3 \cos ^{2} \Phi+\sin (\phi+\Phi) \sin (2 \Phi)\right]\right\},
$$

where $G$ is the striking mass and $M$ is the mass of a shell. The generalised forces are

$$
Q_{\Phi}=-3 M_{p}(\Phi) / 2
$$

The equation of motion of the model in Fig. 7(a) is obtained from the Lagrange's equation

$$
\frac{d}{d t}\left(\frac{\partial T}{\partial \dot{\Phi}}\right)-\frac{\partial T}{\partial \Phi}=Q_{\Phi}
$$

as 


$$
\begin{aligned}
\ddot{\Phi}= & \left\{9 G \sin (3 \Phi)+m \sin (\Phi / 2)\left[\cos \Phi \cos (\Phi / 2)+\left(1-14 \sin ^{2}(3 \Phi / 2)\right)-1\right]+\right. \\
& \left.\left.\frac{\sin \Phi \cos \Phi}{4-\sin ^{2} \Phi}\left(12 G \sin (3 \Phi / 2)^{2}+m\left(3 \cos ^{2} \Phi+\sin (3 \Phi / 2)+1\right)\right)\right] \dot{\Phi}^{2}+\left[18 M_{p}\left(4-\sin ^{2} \Phi\right)\right] /(L / 3)^{2}\right\} \times \\
& {\left[12 G \sin (3 \Phi / 2)^{2}+m\left(3 \cos ^{2} \Phi+\sin (3 \Phi / 2)+1\right)\right]^{-1}, }
\end{aligned}
$$

when substituting $\phi=f(\Phi)$ in Eq.(20). The kinematically admissible rotations at the hinges define $\phi=\sin ^{-1}(0.5 \sin \Phi)$ for $\Phi \in[0, \pi / 2]$. However, a simplification $\phi=\Phi / 2$ is used to obtain Eq. (23), so that this equation can be used only for $\Phi<\pi / 4$ with an acceptable accuracy. In Eq. (23), $M_{p}$ is the plastic bending moment, which takes into account the ovalization of the cross-section of a circular shell, $M_{p}=M_{0}\left(1-0.533 R^{2} \kappa / h\right)\left(1+0.644 R^{2} \kappa / h\right)[20]$, where $M_{0}=\sigma^{0} h D^{2}$ and $\sigma^{0}=\sigma_{0}+E_{h} V_{0} t^{*} / L$. It should be noted that $\sigma^{0}$ can be taken as an average flow stress when an actual stress-strain curve is available. The average curvature can be expressed as $\kappa=\frac{3}{2} \Phi \frac{2}{3 L}=\Phi / L$, therefore

$$
M_{p}(\Phi)=M_{0}\left(1-0.533 R^{2} \Phi /(L h)\right)\left(1+0.644 R^{2} \Phi /(L h)\right)
$$

The initial conditions for the global bending phase are

$$
\Phi(0)=2\left[\varphi_{0}+\varphi\left(t^{*}\right)\right], \quad \dot{\Phi}(0)=2 \dot{\varphi}\left(t^{*}\right)
$$

thereby linking the models for the compression and bending phases for the global bending mode. The total time for the deformation of a tube into a global bending mode defined by the models in Fig. 6(a) and $7(\mathrm{a})$ is $t^{*}+t_{\text {glob }}$, where $t^{*}$ is the duration of the compression phase and $t_{g l o b}$ is the time for the development of large bending deformations described by Eq. (23).

\subsubsection{Progressive buckling}

A model with plastic hinges is used to describe the post-buckling behaviour of a local fold with an axial length $l=2 \tilde{l}$, where $\tilde{l}=(\pi h R / \sqrt{3})^{1 / 2}$ [2], as shown in Fig. 7(b). During this phase of deformation the distal end of the fold at point $\mathrm{C}$ remains stationary. Accordingly, the moments acting on the model produce a variation of work

$$
\delta W=-2 M_{A} \delta \Psi-2 M_{B} \delta \Psi-2 M_{C} \delta \Psi=\delta Q_{1}+\delta Q_{2}+\delta Q_{3}
$$

Hence the generalised forces are

$$
\begin{aligned}
& Q_{\Psi}=-2 M_{A}-2 M_{B}-2 M_{C}, \\
& \delta Q_{1}=-2 M_{0} \delta \Psi, \delta Q_{2}=-M_{0}(1+\tilde{l} \sin \Psi / R) \delta 2 \Psi, \delta Q_{3}=-2 h \tilde{l} \sigma_{0} \cos \varphi \delta \Psi,
\end{aligned}
$$

where $M_{0}=\sigma^{0} h^{2} /(2 \sqrt{3})$. 
The kinetic energy of the model in Fig. 7(b) is

$$
T=\frac{2 m_{1} l^{2}}{3} \dot{\Psi}^{2}+2 l^{2}\left(m_{1}+G_{1}\right) \dot{\Psi}^{2} \sin ^{2} \Psi
$$

where $G_{1}=G / 2 \pi R$ and $m_{1}=\rho h l$.

The equation of motion for the model in Fig. 7(b) is obtained from the Lagrange's equation

$$
\frac{d}{d t}\left(\frac{\partial T}{\partial \dot{\Psi}}\right)-\frac{\partial T}{\partial \Psi}=Q_{\Psi},
$$

when substituting $Q_{\Psi}$ and $T$ according to Eq. (27) and (28), which gives

$$
\ddot{\Psi}=-\frac{\left(G_{1}+m_{1}\right) \dot{\Psi}^{2} \sin \Psi \cos \Psi}{m_{1} / 3+\left(G_{1}+m_{1}\right) \sin \Psi^{2}}-\frac{M_{0}+M_{0}[1+l \sin \Psi /(2 R)]+\sigma_{0} h l^{2} \cos \Psi /(4 R)}{2 l^{2}\left[m_{1} / 3+\left(G_{1}+m_{1}\right) \sin \Psi^{2}\right]}
$$

The initial conditions for the post-buckling deformation of a single fold modelled as shown in Fig. 7(b) are

$$
\Psi(0)=\left[\psi_{0}+\psi\left(t^{*}\right)\right] \quad \dot{\Psi}(0)=\dot{\psi}\left(t^{*}\right)
$$

thereby linking the models for the compression and bending phases for the progressive buckling mode. The total time for the deformation of a tube into a progressive buckling mode defined by the models in Fig. 6(b) and 7(b) is $t^{*}+t_{\text {progr }}$, where $t^{*}$ is the duration of the compression phase and $t_{\text {progr }}$ is the time for the development of large bending deformations described by Eq. (30).

\section{Analysis of the models' behaviour}

In this section, the responses of the proposed structural models are verified by comparing them with the force-time histories and the final deformed shapes predicted by the numerical finite-element simulations introduced in section 2. An analysis of the imperfection sensitivity of the analytical models is presented in Appendix 1. Unified initial imperfections, such as $\varphi_{0}=\psi_{0}=0.0015$, which give small associated lateral displacements $w_{0}$ and $W_{0}$, are used in all the subsequent examples in this paper. The same magnitude of the initial imperfections was used in the numerical simulations to calibrate the FE model shown in Fig. 1 with the experimental results.

\subsection{Initiation of buckling}

As remarked in Section 3, the genesis of the buckling modes occurs during the compression phase and are related strongly to the characteristics of this phase, such as the relative duration and vertical velocities at $t=t^{*}$, which are influenced by the impact velocity, yield stress and strain hardening, as well as the shell geometry. 
The variation of the vertical velocities at the proximal ends of the models in Fig. 6 due to the rotation of their links, $V_{1, \text { glob }}$ and $V_{1, p r o g r}$, according to Eq.(2) and Eq. (19), respectively, together with the variation of the velocity of the striker, $V_{\text {str }}$, determined by Eq.(3), are shown in Fig. 8. The durations of the compression phases associated with the two buckling modes $t_{\text {glob }}^{*}$ and $t_{\text {progr }}^{*}$ are marked in this figure. If the models' behaviour is related to the response of an actual tube, one can anticipate that the mode, which grows at a greater rate, will develop with increasing displacements and interrupt the compression phase of the tube. Thus, the end of the compression phase of a tube can be defined as

$$
t^{*}=\min \left(t_{g l o b}^{*}, t_{\text {progr }}^{*}\right) \text {. }
$$

Let us assume that the first fold forms near to the proximal end of a circular shell if progressive buckling is initiated, otherwise, the tube responds by global bending. If the compression capacity related to the progressive buckling mode is exhausted earlier than the corresponding capacity for the global bending mode, then because the striker maintains contact with the proximal end of the tube the associated velocity is

$$
V_{\text {str }}\left(t^{*}\right)=V_{1, p r o g r}\left(t^{*}\right), \quad V_{1, g l o b}\left(t^{*}\right)<V_{\text {str }}\left(t^{*}\right)
$$

if not

$$
V_{\text {str }}\left(t^{*}\right)=V_{1, g l o b}\left(t^{*}\right), \quad V_{1, p r o g r}\left(t^{*}\right)<V_{s t r}\left(t^{*}\right) .
$$

According to the definitions given by Eqs (32) and (33), a higher vertical velocity $V_{1}$ is associated with the leading mode. One can see in Fig. 8(a) that according to the models' predictions, the global bending mode develops more rapidly than progressive collapse for a low velocity impact, which can result in final global bending of a tube. The buckling shape obtained from the numerical simulations for these loading conditions is shown in the same figure. A higher impact velocity causes a local fold to develop more rapidly than a global bending mode as shown in Fig. 8(b) (The numerical predictions for the final buckling shape is also shown in this figure). The rotation angles obtained at $t=t^{*}$ for the two cases of loading are $\varphi\left(t^{*}\right)=0.086 \mathrm{rad}, \psi\left(t^{*}\right)=0.0411 \mathrm{rad}$ at $t^{*}=t^{*}{ }_{g l o b}=1.052 \mathrm{msec}$ for the $6 \mathrm{~m} / \mathrm{s}$ impact while $\varphi\left(t^{*}\right)=0.0182 \mathrm{rad}, \psi\left(t^{*}\right)=0.122 \mathrm{rad}$ at $t^{*}=t^{*}$ progr $=0.839 \mathrm{msec}$, for the impact with $8.5 \mathrm{~m} / \mathrm{s}$. The corresponding velocities calculated from the models are $\dot{\varphi}\left(t^{*}\right)=155 \mathrm{rad} / \mathrm{s}, \dot{\psi}\left(t^{*}\right)=103.7$ $\mathrm{rad} / \mathrm{s}$ for the $6 \mathrm{~m} / \mathrm{s}$ impact, but $\dot{\varphi}\left(t^{*}\right)=58.4 \mathrm{rad} / \mathrm{s}, \dot{\psi}\left(t^{*}\right)=147.5 \mathrm{rad} / \mathrm{s}$ for the impact with $V_{0}=8.5 \mathrm{~m} / \mathrm{s}$. It appears that the more rapid formation of a local fold when increasing the impact velocity is the major mechanism for the stabilisation of a long tube (i.e., development of progressive buckling).

The influence of the material properties on the duration of the compressive phase is seen from the comparison between Fig. 8(b) and 8(c) showing the models' responses for Mat1 and Mat3 for an impact velocity of $8.5 \mathrm{~m} / \mathrm{s}$. An increase of the duration of the compression phases for both buckling modes is observed for the material with the lower yield stress, while a larger increase is seen for the 
time to form a local fold $\left(t_{\text {progr }}^{*}\right)$. These effects influence the associated vertical velocities $V_{1, \text { glob }}$ and $V_{1, \text { progr }}$.

The influence of the tube length on the duration of the compression phases of shells, with $D=50.2$ $\mathrm{mm}, h=2.19 \mathrm{~mm}$ made from Mat4 and subjected to identical impact loadings, $V_{0}=9.6 \mathrm{~m} / \mathrm{s}$ and $\mathrm{G}=$ $209 \mathrm{~kg}$, is shown in Figs. 9(a) and 9(b), as predicted by the models in Fig. 6. It is observed that the increase of the tube length leads to compression phases having longer durations for both buckling modes, which is consistent with the force-time histories presented in Figs. 5(a) and 5(b). In addition, it is observed that the compression phases for both modes have similar durations, which become equal for $L=750 \mathrm{~mm}$. In this case, the global and local buckling modes start to form simultaneously at $t=t^{*}$ and can continue to develop at comparable rates during the subsequent deformation phase. Thus, an unpredictable response of an actual tube can be anticipated in this case. A further increase of the impact velocity separates the two buckling modes since the duration of the compressive phase for the progressive buckling mode decreases, as shown in Fig. 9(c).

\subsection{Post-buckling behaviour}

It was observed from the models' behaviour, which describe the pre-buckling response of an actual shell, that the initial conditions for the subsequent bending/buckling phases at $t=t^{*}$, in combination with the material properties of a shell, cause a significant effect on the formation of the corresponding post-buckling mode since they determine the development rate of the primary buckling modes.

An illustration of the model responses for the post-buckling behaviour, either progressive buckling or global bending, is shown in Fig 10. In these figures, the curves are shifted an amount equal to the duration of the corresponding compression phase $t^{*}$. It is evident that global bending described by the model in Fig. 7(a) develops faster than the formation of a local fold for an impact with $V_{0}=6 \mathrm{~m} / \mathrm{s}$ (Fig. 10(a)). By way of contrast, the variation of the rotational angles in Fig. 10(a) indicate a much more rapid development of a local fold for $V_{0}=8.5 \mathrm{~m} / \mathrm{s}$. The different responses in Fig. 10(a) arise from the corresponding initial conditions. At $t=t^{*}$, the second phase of deformation for $V_{0}=6 \mathrm{~m} / \mathrm{s}$ (Fig. 8(a)) starts to develop with a larger rotational angle and velocity associated with the global bending mode. For an impact with $V_{0}=8.5 \mathrm{~m} / \mathrm{s}$ (Fig. 8(b)), the duration of the compressive phase is determined by the local buckling mode so that at $t^{*}=t_{\text {progr }}^{*}$ the initial conditions for the global mode are $\Phi(0)=0.0394 \mathrm{rad}$ and $\dot{\Phi}(0)=116.8 \mathrm{rad} / \mathrm{s}$, while the corresponding conditions for the $6 \mathrm{~m} / \mathrm{s}$ impact are $\Phi(0)=0.0852 \mathrm{rad}$ and $\dot{\Phi}(0)=310 \mathrm{rad} / \mathrm{s}$. 
The variation of the characteristic angles $\Phi$ and $\Psi$ in Fig. 10(a) implies that the leading mode at the end of the compression phase continues to grow at a higher rate during the buckling/bending phase, i.e., the buckling mode of a tube cannot alter during this phase.

The influence of the material properties on the development of the two buckling modes (global and progressive) is shown in Fig. 10(b). It is evident that a global collapse (Eq.(30)) develops more rapidly for materials having a lower yield stress due to the smaller bending rigidity associated with the ovalisation of the cross-section. The compression phase for this example is associated with the parameters in Fig. 8(c). The local folds grow at similar rates for the two materials. The bending rigidity for the model with the characteristics of Mat3 is smaller but the bending phase starts at a lower velocity than for the model with Mat1 which leads to the observed response.

\section{Parametric analysis of buckling transition}

In this section, a quantitative analysis of the influence of the tube and loading characteristics on the buckling transition is presented. The duration of the compression phases of the two principal buckling modes are compared in order to analyse the influence of the parameters on the variation of the vertical velocity at the end of this phase.

The tube length is of primary interest for the buckling transition in this study. The predicted durations of the compression phases for the global bending and progressive buckling modes are presented in Fig. 11 as functions of the impact velocities for tubes with $D=50.8 \mathrm{~mm}, h=2 \mathrm{~mm}$ and made from Mat1. In this figure, the impact velocity $V_{0, t}$ is defined at $t^{*}=t_{\text {glob }}^{*}=t_{\text {progr }}^{*}$ and corresponds to the lowest impact velocity that can cause a transition from global bending to progressive buckling. However, if this velocity becomes independent of the tube length for tubes longer than certain length (see also Fig (9b)), then $V_{0, t}$ is referred to as an impact velocity $V_{0, c i}=V_{0, t}$ which causes a counter-intuitive behaviour of a long tube. For example, $V_{0, t}$ increases only by 0.02 $\mathrm{m} / \mathrm{s}$ when the tube length increases from $650 \mathrm{~mm}$ to $750 \mathrm{~mm}$ in Fig. 11. Thus, the durations of the compressive phases associated with the global and progressive buckling modes remain comparable for a wide range of the tube lengths $L>650 \mathrm{~mm}$. Therefore, the impact velocity of $7 \mathrm{~m} / \mathrm{s}$ can be regarded as $V_{0, c i}$ for this particular tube cross-section.

This phenomenon can occur in any dynamically loaded cylindrical shell, but the impact velocity $V_{0, c i}$ depends on the material and geometrical properties of the shell. Numerical finite-element predictions for the buckling shapes of circular tubes with $h=2.19 \mathrm{~mm}, D=50.2 \mathrm{~mm}$ and made from material Mat4, which exhibit a counter-intuitive response for an impact loading with $G=209 \mathrm{~kg}$ and $V_{0}=10.4 \mathrm{~m} / \mathrm{s}$, are presented in Fig. 12(a). The calculated duration of the compression phases from the analytical models shows that a vertical impact velocity $V_{0, c i}=9.6 \mathrm{~m} / \mathrm{s}$ (which is somewhat lower than 
the velocity in the numerical simulations) causes $t_{\text {glob }}^{*}=t_{\text {progr }}^{*}$ for a large range of tube lengths. The durations of the compression phases for the global and progressive buckling modes remain very similar for tube lengths between $550 \mathrm{~mm}$ and $750 \mathrm{~mm}$, approximately, as shown in Fig. 12(b). A counter-intuitive response was also observed experimentally for aluminium alloy tubes having the same cross-sectional dimensions and subjected to an impact loading with $G=209 \mathrm{~kg}$ and $V_{0}=10.4$ $\mathrm{m} / \mathrm{s}$ [8]. The stress-strain curve of the material for the test tubes was linearised to obtain Mat4.

The static buckling transition is governed by the ratio $D / h$. This ratio also affects the initiation of the dynamic buckling transition. The duration of the compression phase increases with an increase of the tube thickness thereby requiring a higher impact velocity to cause a transition to progressive buckling (Fig. 13(a)) in a tube having a constant length. In other words, for the same impact velocity, a thicker tube has a shorter critical length. The analytical models predict in Fig. 13(b) the relative variation of the vertical velocities $V_{1, \text { glob }}\left(t^{*}\right)$ and $V_{1, \text { progr }}(t)$ at the proximal ends of tubes having identical lengths and diameters made from Mat4 and subjected to axial impact loadings with $\mathrm{G}=209$ $\mathrm{kg}$. In this figure, the vertical velocities $V_{1}\left(t^{*}\right)=f\left(V_{0}\right)$ are combined in a way that

$$
\begin{array}{llll}
V_{1, \text { glob }}\left(t^{*}\right)=V_{1, \text { glob }}\left(t_{\text {glob }}^{*}\right), & V_{1, \text { prog }}\left(t^{*}\right)=V_{1, \text { progr }}\left(t_{\text {glob }}^{*}\right) & \text { for } & t_{\text {glob }}^{*} \leq t_{\text {progr }}^{*}, \\
V_{1, \text { glob }}\left(t^{*}\right)=V_{1, \text { glob }}\left(t_{\text {progr }}^{*}\right), & V_{1, \text { prog }}\left(t^{*}\right)=V_{1, \text { progr }}\left(t_{\text {progr }}^{*}\right) & \text { for } & t_{\text {glob }}^{*} \geq t_{\text {progr }}^{*}
\end{array}
$$

to show the variation of both velocities (global and progressive) at the end of compression phase defined by Eq. (32). One can see in Fig. 13(c) that for lower impact velocities, the vertical velocity associated with the progressive mode is slightly lower that the one corresponding to a global buckling mode (i.e. global bending occurs). By way of contrast, the vertical velocity for the global mode decreases rapidly, when conditions for progressive buckling occur for higher impact velocities (Fig. 13(b)). Therefore, the relative variation of the vertical velocities of the two buckling modes at the end of the compression phase can be considered a key factor for the development of the dominant mode during the subsequent large deformation phase.

An increase of the hardening modulus causes an increase in the duration of the compression phases for both principal modes, but it affects more significantly the progressive buckling mode, as shown in Fig. 14 which compares two materials having an equal yield stress but different $E_{h}$ (Mat1 and Mat2). An increase of the impact velocity is required to initiate a buckling transition in the tube made from the material with the larger strain hardening modulus (Mat2).

It should be emphasized, however, that an increase of the transition velocity $V_{0, t}$ is not always related to the increase of the duration of the compression phase. Unlike the previous examples, an increase of the yield stress for the same ratio $E_{h} / \sigma_{0}=1.7$ (Mat1 and Mat4) leads to a decrease of the duration of the compression phase for both principal modes, but it affects more significantly the global bending, as shown in Fig, 15(a). The higher yield stress causes a more rapid development of the global 
mode during compression (see Eq. (2)), while the development of the local fold is only slightly affected. Therefore, a higher impact velocity is required to initiate progressive buckling in the tube made from the material with the higher yield stress (Mat4), as evident in Fig. 15 (b). The values of $\psi\left(t^{*}\right)$ for models with Mat4 are smaller than the corresponding angles for Mat1. The variations of the characteristic angles $\psi\left(t^{*}\right)$ and $\varphi\left(t^{*}\right)$ with the impact velocity are shown in Fig. 15(c) for the two materials.

It is important to note that while the impact velocity predominantly governs the initial buckling transition, the striking mass influences the final buckling shape as well, as shown in Fig. 16(a). If the impact energy is increased by increasing the striking mass then the initial deformation pattern remains unchanged. However, the gradual increase of the lateral displacements associated with global bending (Figs. 16(b)) reaches a larger magnitude for a larger impact mass. According to the analytical models in this paper, the characteristic angles for the global bending mode at the end of the compression phases $-\varphi\left(t^{*}\right)=0.0438 \mathrm{rad}(\Phi(0)=0.0906 \mathrm{rad})$ for an impact with a mass of $500 \mathrm{~kg}$, are slightly larger than the values $\varphi\left(t^{*}\right)=0.0423 \mathrm{rad}(\Phi(0)=0.0867 \mathrm{rad})$ for a $209 \mathrm{~kg}$ mass impact. Although, initially, there is only a marginal difference between the bending angles $\Phi$ (Fig. 16(c)), the global transverse displacements accumulate after the formation of each subsequent fold. Therefore, a tube that responds by a progressive buckling initially can switch to a global collapse after the development of some progressive folding as illustrated in Fig. 16(b).

\section{Lower and upper bounds to the impact velocity for the buckling transition}

The observed responses of circular tubes show that an initial buckling pattern in a progressive buckling mode cannot be used as a criterion for progressive buckling throughout the entire response. A gradual increase of the lateral displacements after the formation of each additional fold can cause a switch to global bending depending on the magnitude of the striking mass (Fig. 16(a) and 16(b)). Nevertheless, the observed relations between the type of buckling and the loading parameters (impact velocity and striking mass) suggest that energy based empirical estimates can be obtained to provide lower and upper bounds to the impact velocity causing a buckling transition. The transition velocity $V_{0, t}$ defined in Section 6 can be regarded as a lower bound. It is the maximum impact velocity, which causes a pure global bending of a tube with a given geometry when this tube cannot absorb any external energy by wrinkling. An upper bound to the impact velocity causing a buckling transition is taken as the minimum impact velocity, which causes a classical progressive buckling response. In other words, progressive buckling can be initiated when

$$
V_{1, \text { glob }}\left(t^{*}\right)<V_{1, \text { progr }}\left(t^{*}\right)
$$

at the end of the compression phase at $t=t^{*}$. Inequality (34) can be regarded as a necessary and sufficient condition for the development of progressive buckling if the impact energy is sufficient for 
the development of a single fold only. For impact energies greater, or equal to, the energy sufficient for the development of a single fold, the inequality

$$
V_{1, \text { glob }}\left(t^{*}\right)>V_{1, \text { progr }}\left(t^{*}\right)
$$

determines a global bending response. Together with this, the development of the mixed-mode response shows that the inequality (34) is not a sufficient condition for a larger impact energy. An additional condition, such as a further limitation on the speed $V_{1, \text { glob }}\left(t^{*}\right)$, is necessary in order to prevent the deformation from switching into a global bending mode.

The deformation of a shell made from a ductile material and subjected to an axial impact loading can be related to its energy absorption capacity [3] and, in particular, to the energy dissipated during the development of a single fold, $T_{p, f}$ (see Appendix 2). An initial impact velocity, which is sufficient to cause the development of a single fold, can be defined as

$$
V_{s f}=\sqrt{2 T_{p, f} / G}
$$

where $G$ is the striking mass. The axial velocity at the proximal end of shells subjected to larger impact energies, which can potentially produce more folds in the case of progressive buckling, decreases almost linearly during the response. Under the above assumption, each subsequent fold starts to develop at a lower vertical velocity in comparison with the former one. Examples for the vertical velocity at the proximal end of a tube at the initiation of successive folds are shown in Appendix 2, Fig. A2.3 for a constant impact energy (Fig. A2.3(a)) and two different striking masses (Fig. A2.3(b)) using the following relationship

$$
\left(V_{1, k}^{2}-V_{1, k+1}^{2}\right) G / 2=T_{p, f}, \quad k=1, \ldots, n-1
$$

where $V_{1,1} \approx V_{0}$. It turns out that the minimum vertical velocity at the start of the development of the fold depends on the striking mass and is equal to $V_{s f}$.

On the other hand, the global bending mode of a tube can also potentially continue to grow with an initial vertical velocity $V_{1, k+1}$ after the formation of the $k^{\text {th }}$ fold, as discussed previously. As the most restrictive condition it can be required that

$$
V_{1, g l o b}\left(t^{*}\right)<V_{s f}
$$

in order to satisfy $V_{1, \text { glob }}<V_{1, \text { progr }}$ at the initiation of the development of each subsequent fold although, in general, $V_{s f}<V_{1, k}$. According to inequality (38), the limit of the vertical velocity, $V_{1, \text { glob }}\left(t^{*}\right)$, associated with the global bending mode, is related to striking mass. Therefore, $V_{1, \text { glob }}\left(t^{*}\right)$ must decrease when increasing the impact energy by increasing the impact mass in order to maintain the progressive folding of a shell, so that 


$$
V_{1, \text { glob }}\left(t^{*}\right)<\min \left\{V_{s f}, V_{1, \text { progr }}\left(t^{*}\right)\right\}
$$

becomes a sufficient condition for a stable response (progressive buckling) of a long circular shell under an axial impact. For a convenience, ratio $V_{s f} / V_{0}=\eta, 0<\eta<1$, is introduced and the above inequality becomes

$$
V_{1, \text { glob }}\left(t^{*}\right)<\min \left\{\eta V_{0}, V_{1, p r o g r}\left(t^{*}\right)\right\}, \quad \eta<1 .
$$

It is anticipated that, for a relatively large striking mass, the deceleration of the mass during the compression phase is small and, therefore, $V_{1, \text { progr }}\left(t^{*}\right) \approx V_{0}$ in the case of initiation of a progressive buckling response. If $T_{0}>T_{p . f}$ and $\min \left\{\eta V_{0}, V_{1, \text { progr }}\left(t^{*}\right)\right\}=V_{\text {sf }}$, the impact velocity, which satisfies inequality (38), is

$$
V_{0}>V_{1, g l o b}\left(t^{*}\right) / \eta \text {. }
$$

The minimum impact velocity, which satisfies inequality (40), is called an 'upper bound' and the tube responds entirely by progressive buckling. A tube will respond to an impact velocity by global bending only if the inequality (35) is satisfied. Thus, inequalities (35) and (40) determine the lower and upper bounds to the impact velocity, respectively, for the buckling transition of long circular tubes subjected to axial impact loadings. The upper bound to the impact velocity can be regarded as a 'critical impact velocity', $V_{0, c r}$, which causes a classical progressive buckling of a circular tube. Impact loadings, which produce vertical velocities $V_{1, g l o b}\left(t^{*}\right)$ within the interval

$$
\eta V_{0, c r}<V_{1, g l o b}\left(t^{*}\right)<V_{1, p r o g}\left(t^{*}\right)
$$

will cause a mixed mode response.

The criterion for a dynamic buckling transition can be expressed with respect to the vertical velocity associated with a global bending mode as

$$
V_{1, g l o b}\left(t^{*}\right)=3 \gamma \varphi_{0}^{2} L \sinh \left(2 \gamma t^{*}\right) \leq\left(2 T_{p, f} / G\right)^{1 / 2},
$$

where the duration of the compression phase determined by the progressive mode $t^{*}=t_{\text {progr }}^{*}<t_{\text {glob }}^{*}$ is calculated using Eqs. (2), (3), (17-19) and (32).

One can see that condition (42) for the dynamic buckling transition when applied to a tube with given material and geometric characteristics depends on both the initial impact velocity (through the duration of the compression phase) and the striking mass. Larger impact masses impose a requirement for a lower $V_{1, g l o b}\left(t^{*}\right)$, which can promote a classical progressive collapse, while a decrease of the striking masses relaxes the limitation for $V_{1, \text { glob }}\left(t^{*}\right)$.

Let us consider impact loadings with a constant initial kinetic energy applied to circular tubes with $D=50.8 \mathrm{~mm}, h=2 \mathrm{~mm}$ and made from Mat1. The velocity ranges for the development of the buckling modes of tubes, which have two different lengths and are subjected to impacts with a 
constant initial kinetic energy of $5 \mathrm{~kJ}$ (Mat1) are shown in Fig. 17(a) according to Eqs. (34) and (42) when using $T_{p, f}=1000 \mathrm{~J}$. The line $V_{1}=V_{0}$ in this figure is shown to illustrate the difference between the initial axial velocity and the velocity of the striker at the end of the compression phase. As expected, the impact velocities, which correspond to both velocity bounds, $V_{0, t}$ and $V_{0, c r}$, increase when increasing the tube length. For example, an axial impact with $V_{0}=7.5 \mathrm{~m} / \mathrm{s}$ is predicted to cause progressive buckling of a tube having $L=550 \mathrm{~m}$, while a $650 \mathrm{~mm}$ long tube is expected to buckle into a mixed mode. Since smaller impact masses correspond to higher impact velocities for a constant impact energy, the limitation on the vertical velocity, $V_{1, g l o b}\left(t^{*}\right)=\left(2 T_{p, f} / G\right)^{1 / 2}$, appears as an increasing function of the impact velocity as shown in Fig. 17(a). This result shows that higher velocity impacts can lead to a relatively rapid stabilization of the tube response, particularly for low impact energies, which produce a constant but small number of folds, which develop at a faster rate. This explains the observation in some experimental tests on tubes subjected to a low impact energy that a significant increase of the critical length occurs for the buckling transition when increasing the impact velocity [8].

It can be shown that the upper bound to the impact velocity, $V_{0, c r}$, can increase significantly for impact loadings with large energies associated with large striking masses. The velocity ranges for the development of the buckling modes, for the same tubes with $L=650 \mathrm{~mm}$ and subjected to impact loadings with $G_{1}=200 \mathrm{~kg}$ and $G_{2}=500 \mathrm{~kg}$, are shown in Fig. $17(\mathrm{~b})$. In this case, $V_{1, g l o b}\left(t^{*}\right)=$ $\left(2 T_{p, f} / G\right)^{1 / 2}=$ const for each striking mass and is independent of the impact velocity. This result suggests that the tube response can be stabilized (to develop classical progressive buckling) only when increasing significantly the impact velocity. Figs 17 (a) and 17(b) imply that different critical tube lengths can be expected at the buckling transition for different impact energies.

A comparison between the numerically obtained critical tube lengths at the buckling transition for a constant impact energy of $5 \mathrm{~kJ}$ [9], and the predictions from the simplified models are shown in Fig. 18(a). The curve marked as ' 1 ' shows the predicted critical lengths according to condition (34), which is a necessary condition for a transition. One can see in this figure that around an impact velocity of $7 \mathrm{~m} / \mathrm{s}$, the models predict a significant increase of the tube length for a small variation of the impact velocity. This impact velocity, in fact, is associated with a velocity $V_{0, c i}$, which causes a counter-intuitive response of a tube for the particular cross-sectional geometry and material properties. The critical lengths at a buckling transition presented by curve ' 2 ' are obtained from Eq. (42) for impacts with a constant energy of $5 \mathrm{~kJ}$. It shows that the actual number of folds for a given tube length requires a higher impact velocity (according to Eq. (42)) then the one corresponding to the initiation of progressive buckling. Curve ' 3 ' in Fig. 18(a) is obtained from Eq. (42) when considering impact loadings with a constant mass of $500 \mathrm{~kg}$. The theoretical predictions in Fig. 18(a) are in qualitative agreement with the numerical results showing a correct trend, although the analytical models predict a 
somewhat more rapid stabilization at higher impact velocities. A partial explanation for this behaviour can be attributed to the development of asymmetric folds in the actual tube at higher impact velocities, which contribute to an increase of the tube eccentricity. Comparison between curves ' 2 ' and ' 3 ' shows that an increase of the impact energy (for higher impact velocities) leads to a further increase of the critical velocity, which is required to cause a classical progressive buckling mode. One can see that the critical velocity at a buckling transition of a tube having a constant length increases when increasing the striking mass. (The critical lengths along curve '2' correspond to decreasing impact masses and $G<500 \mathrm{~kg}$ for $V_{0}>4.47$.) Therefore, it is possible that for a sufficiently large striking mass no significant increase of the critical tube length for a buckling transition can be achieved, in comparison with the static case, regardless of any increase of the impact velocity.

As mentioned in Sections 5 and 6, the strain hardening modulus of the shell material also influences the relative variation of the velocities $V_{1, \text { glob }}\left(t^{*}\right)$ and $V_{1, \text { progr }}\left(t^{*}\right)$ (see also Fig. 14), which affects the critical impact velocity for a buckling transition. Figure 18(b) shows a comparison between the predictions of the models and the numerical results obtained in Ref. [9] for a constant impact energy of $5 \mathrm{~kJ}$ on tubes with $D=50.8 \mathrm{~mm}, h=2 \mathrm{~mm}$ and made from two materials having the same yield stress but different strain hardening moduli. It is observed that a tube made from the material with a lower strain hardening characteristic (Mat1) responds by a classical progressive buckling to lower initial impact velocities than for tubes made from a material with a higher strain hardening modulus (Mat2). The models predict a faster stabilization at higher impact velocities as observed in the previous example.

According to Eq. (42), the variation of the yield stress can have an effect not only on the speed of the formation of the buckling modes, as discussed earlier, but it also will enter into the condition for the limitation of $V_{1, g l o b}\left(t^{*}\right)=\left(2 T_{p, f} / G\right)^{1 / 2}$. For example, shells made of materials with a low yield stress should develop a larger number of folds to absorb a certain impact energy than tubes made from material with a higher yield stress. Thus, a more restrictive condition will apply to $V_{1, \text { glob }}\left(t^{*}\right)$, which results in an increase of the velocity range for the mixed mode and an increase of the critical impact velocity to obtain classical progressive buckling.

It should be mentioned that velocity limit $V_{1, g l o b}\left(t^{*}\right)=\left(2 T_{p, f} / G\right)^{1 / 2}$ varies with the tube geometry too, and, in particular, with the thickness $h$ through the plastic energy $T_{p, f}$. According to Eq. (42), the critical impact velocity may increase when decreasing the tube thickness, particularly for high energy impact loadings with a large striking mass. A larger number of folds is required for a thinner tube to absorb a given impact energy, which causes larger lateral displacements in the global bending mode to accumulate after the formation of each fold. In this case, it is possible that $V_{1, g l o b}\left(t^{*}\right)$ for the thinner tube will become larger than $V_{1, g l o b}\left(t^{*}\right)$ for the thicker tube. Thus, the tube length, which promotes a 
classical progressive response to a certain impact velocity, may not increase when decreasing $h$, which is in contrast to the static loading case [4]. This type of response was observed by Jensen et al. [21,22] in their experiments on long tubes having square cross sections and made from an aluminium alloy and subjected to axial impact loadings having a constant initial kinetic energy. It was reported in [22] that the critical tube length, which promotes a classical progressive collapse for $V_{0}=13 \mathrm{~m} / \mathrm{s}$, increases for wall thicknesses $3.5 \mathrm{~mm}$ and $4.5 \mathrm{~mm}$, but decreases for $h=2.5 \mathrm{~mm}$ and $2.0 \mathrm{~mm}$.

This phenomenon cannot be explained directly by inertia effects since the decrease of the wall thickness promotes a more rapid development of the local folds, which causes a stabilisation of the tube. Thus, it is possible that despite the stabilizing effect of the local wrinkling, a tube with a smaller wall thickness will require a larger critical impact velocity $V_{0, c r}$ and a mixed collapse mode will be observed within a larger range of the impact velocities.

\section{Concluding remarks}

The transition conditions between a progressive buckling mode and a global bending collapse of axially impacted cylindrical shells are studied when estimating the formation times for the principal buckling modes that exist in long circular tubes which buckle plastically. Structural models are proposed, which take into account the characteristic features of the two deformation phases: axial compression and post-buckling response. The behaviour of the models, which describe the compression phase, provides an insight into the mechanism of the buckling transition. The dominant buckling mode is characterised by a larger vertical velocity at the end of the compression phase, which leads to a rapid development of this mode during the post-buckling response. There is, however, an impact velocity associated with a given set of geometrical and material parameters, which causes a simultaneous increase of the progressive buckling and global bending modes, so that no 'leading' mode can be selected for buckling. In this case, both buckling modes can start to develop rapidly during the post-buckling deformation phase making it difficult to predict the development of a particular collapse mode. This can lead to a counter-intuitive response, which has been observed in experimental studies and numerical simulations.

An empirical criterion, which depends on the impact energy, is used to predict the lower and upper bounds to the critical impact velocity for a buckling transition. This criterion is based on the energy absorbed during the development of a single fold and the vertical velocities at the proximal end of a shell at the end of the compression phase, which are associated with the two principal collapse modes (global bending or progressive buckling).

The present study reveals that it is not possible to draw simple charts for the critical tube length associated with a buckling transition similar to the ones presented in Refs. [4] and [5] or any simple curve-fit dependence. Such dependences should be shown in a multi-dimensional space of parameters, which influence the buckling transition, namely the shell geometry, material properties, impact 
velocity and impact energy. Nevertheless, this study shows that the dynamic buckling transition is not a random phenomenon when all the parameters, which influence the process, are known. Moreover, it is demonstrated that the variation of each parameter influences the tube response in a predictable manner.

The analysis of the buckling process of relatively long circular tubes presented in this paper suggests that an introduction of an appropriate trigger (either mechanical dent or altering locally the material properties) can improve the energy absorbing characteristics of a long tube by promoting the initiation of a progressive buckling mode. However, in the case of a large impact energy, which requires a large number of folds to absorb the initial kinetic energy, the buckling initiation into the desirable buckling mode might change later from progressive buckling into a mixed buckling mode, which includes global bending. Therefore, the upper bound to the impact velocity is referred to as a 'critical impact velocity', which causes a classical progressive collapse of a circular tube with a given geometry when subjected to an impact with a known initial kinetic energy.

The present study counsels that, for practical purposes, long tubes subjected to an axial impact should be analysed for the actual striking mass. The velocities causing comparable initial conditions for the buckling/bending phases and, therefore, counter-intuitive deformation of the energy absorbers, can be avoided by a judicious variation of the cross-sectional characteristics of the tubes. If a certain geometry is required, different materials can be selected to assure a predictable response of the energy absorber.

Acknowledgement - The support from the Bulgarian National Research Fund through contract TH-1103/01 and from the Engineering and Physical Sciences Research Council through grant GR/M750044 is acknowledged. 


\section{References}

1. Alghamdi AAA. Collapsible impact energy absorbers: an overview. Thin-Walled Structures 2001;39:189-213.

2. Alexander JM. An approximate analysis of the collapse of thin cylindrical shells under axial load. Quarterly J Mechanics and Appl Math 1960;13:10-15.

3. Jones N. Structural Impact. Cambridge University Press, Cambridge 1989. (Paperback edition, 1997).

4. Andrews KRF, England GL, Ghani E. Classification of the axial collapse of circular tubes under quasistatic loading. Int J Mech Sci 1983;25:687-696.

5. Abramowicz W, Jones N. Transition from initial global bending to progressive buckling of tubes loaded statically and dynamically. Int J Impact Eng 1997;19(5-6):415-437.

6. Alves M, Karagiozova D. Dynamic global and progressive buckling of circular shells under impact loads. In: A.S. Khan and O. Lopez-Pamies, editors, Plasticity, Damage and Fracture at Macro, Micro and Nano Scales, 2002; pp.621-623, Neat Press, Maryland, USA.

7. Jensen O, Langseth M, Hopperstad OS. Transition between progressive and global buckling of aluminium extrusions. In: N. Jones and C. A. Brebbia and A. M. Rajandran, Editors. Structures Under Shock and Impact VII, 2002; pp. 269-277, W.I.T. Press.

8. Hsu, SS, Jones N. Quasi-static and dynamic axial crushing of thin-walled circular stainless steel, mild steel and aluminium alloy tubes. Int J Crashworthiness 2004;9(2):195-214.

9. Karagiozova D, Alves M. Transition from progressive buckling to global bending of circular shells under axial impact - Part I: Experimental and numerical observations. Int J Solids and Structures. 2004;41:1565-1580.

10. Karagiozova D, Alves M. Transition from progressive buckling to global bending of circular shells under axial impact - Part II: Theoretical analysis. Int J Solids and Structures 2004;41: 1581-1604.

11. Guillow SR, Lu G, Grzebieta RH. Quasi-static axial compression of thin-walled circular aluminium tubes Int J Mech Sci 2001;43:2103-2123.

12. Mahmood HF, Paluszny A. Stability of plate-type box columns under crush loading, Computational Methods in Ground Transportation Vehicles, 1982; AMD-Vol. 50, pp. 17-33, Winter Annual Meeting of A.S.M.E.

13. Mazzolani FM. Aluminium alloy structures, Rittman Advanced Publishing Program, Boston (Mass) 1985.

14. Tam LL, Calladine CR. Inertia and strain-rate effects in a simple plate-structure under impact loading. In. J Impact Eng, 1991;11:349-377.

15. Zhang, TG, Yu TX. A note on a 'velocity sensitive' energy-absorbing structure. Int J Impact Eng 1989;8(1):43-51.

16. Karagiozova D, Jones N. Some observations on the dynamic elastic-plastic buckling of a structural model. Int J Impact Eng 1995;16:621-635.

17. Su, XY, Yu TX, Reid SR. Inertia-sensitive impact energy absorbing systems, Part I - Effect of inertia and elasticity. Int J Impact Eng 1995;16:661-672.

18. Karagiozova D, Alves M, Jones N. Inertia effects in axisymmetrically deformed cylindrical shells under axial impact. Int J Impact Eng 2000;24(10):1083-1115.

19. Florence AL, Goodier JN. Dynamic plastic buckling of cylindrical shells in sustained axial compressive flow. Trans. ASME. J Appl Mech 1968;35:80-86.

20. Wierzbicki T, Sinmao MV. A simplified model of Brazier effects in plastic bending of cylindrical tubes. Int J Pressure Vessels 1997;71(1):19-28.

21. Jensen O, Langseth M, Hopperstad OS. Transition from local to global buckling: Quasi-static and dynamic experimental results. In: Proc. 8-th Int. Symposium on Plasticity and Impact Mechanics, IMPLAST 2003, Ed. NK Gupta, 2003; pp.118-125, Phoenix Publishing House, New Delhi.

22. Jensen O, Langseth M, Hopperstad OS. Experimental investigations on the behaviour of short to long square aluminium tubes subjected to axial loading. Int J Impact Eng 2004;30(8-9): 973-1003. 


\section{Figure captions}

Figure 1 Verification of the numerical model; $L=500 \mathrm{~mm}, D=50.8 \mathrm{~mm}, h=2 \mathrm{~mm}$. (a) The first elastic buckling mode used as initial imperfections with magnitude of $0.0005 \mathrm{~L}$; (b, c) $G=200$, progressive buckling mode for $V_{0}=9 \mathrm{~m} / \mathrm{s}$ and global bending mode for $V_{0}=8.7 \mathrm{~m} / \mathrm{s}-\mathrm{FE}$ simulation (b) and experiment (c).

Figure 2 Buckling shapes at transition conditions. (a, b) $L=300 \mathrm{~mm}, T_{0}=5 \mathrm{~kJ}$, Mat1; (c, d) $L=$ $300 \mathrm{~mm}, T_{0}=5 \mathrm{~kJ}$, Mat3; (e, f) $L=450 \mathrm{~mm}, T_{0}=5 \mathrm{~kJ}$, Mat1; (g, h) $V_{0}=10.4 \mathrm{~m} / \mathrm{s}, G=$ $209 \mathrm{~kg}$, Mat4.

Figure $3 L=450 \mathrm{~mm}, D=50.8 \mathrm{~mm}, h=2 \mathrm{~mm}$, Mat2, $T_{0}=5 \mathrm{~kJ}$. (a,b) Displacement-time histories at the tip of the local folds ( $\mathrm{t}$ - proximal end, $\mathrm{b}$ - distal end) and at the 'bending hinge' $-\mathrm{m}$. (a) $V_{0}=8.5 \mathrm{~m} / \mathrm{s}$; (b) $V_{0}=8.75 \mathrm{~m} / \mathrm{s}$; (c) Force-time histories, $V_{0}=$ $8.5 \mathrm{~m} / \mathrm{s}$ ('g' - global bending), $V_{0}=8.75 \mathrm{~m} / \mathrm{s}$ ('p'- progressive buckling).

Figure 4 Principal deformation modes of a circular cylindrical shell buckled plastically; $L=450$ $\mathrm{mm}, D=50.8 \mathrm{~mm}, h=2 \mathrm{~mm}$ (the models are not to scale). (a) Global bending; (b) Progressive buckling.

Figure 5 Force-time histories at the proximal end for shells made from Mat4, $h=2.19 \mathrm{~mm}, D=$ $50.2 \mathrm{~mm}$ and the corresponding buckling shapes; $G=209 \mathrm{~kg}$. (a) $V_{0}=10.4 \mathrm{~m} / \mathrm{s}$ - global bending collapse; (b) $V_{0}=13.4 \mathrm{~m} / \mathrm{s}$ - progressive buckling and mixed mode collapse; (c) $L=550 \mathrm{~mm}$, progressive buckling.

Figure 6 Simple models for the axial compression phases. (a) Global bending mode; (b) Progressive buckling mode (local fold).

Figure 7 Hinge mechanisms for the post-buckling development. (a) Global bending mode, $\Phi^{*}=\Phi(0) ;\left(\right.$ b) Progressive buckling mode (local fold), $\Psi^{*}=\Psi(0)$.

Figure 8 Compressive phases and final buckling phases, $L=450 \mathrm{~mm}, D=50.8 \mathrm{~mm}, h=2 \mathrm{~mm}$, $T_{0}=5 \mathrm{~kJ}$. (a) $V_{0}=6 \mathrm{~m} / \mathrm{s}$, Mat1; (b) $V_{0}=8.5 \mathrm{~m} / \mathrm{s}$, Mat1; (c) $V_{0}=8.5 \mathrm{~m} / \mathrm{s}$, Mat3.

Figure 9 Compressive phases associated with the global bending and progressive buckling modes for shells with $D=50.2 \mathrm{~mm}, h=2.19 \mathrm{~mm}$, Mat4, $G=209 \mathrm{~kg}$; (a) $L=550 \mathrm{~mm}$, $V_{0}=9.6 \mathrm{~m} / \mathrm{s}$; (b) $L=750 \mathrm{~mm}, V_{0}=9.6 \mathrm{~m} / \mathrm{s}$; (c) $L=550 \mathrm{~mm}, V_{0}=13.4 \mathrm{~m} / \mathrm{s}$.

Figure 10 Variation of the characteristic angles during bending/buckling phases of a shell with $L$ $=450 \mathrm{~mm} D=50.8 \mathrm{~mm}$ and $h=2 \mathrm{~mm}$; Angle $\Phi$ characterises the global bending mode and $\Psi$ describes progressive buckling. (a) Influence of the impact velocity, Mat1; (b) Influence of the material properties, $V_{0}=8.5 \mathrm{~m} / \mathrm{s}$, Mat 1 and Mat 3 .

Figure 11 Duration of the compressive phases, $t^{*}$, associated with a global bending and progressive buckling depending on the impact velocity for several tube lengths; $D=$ $50.8 \mathrm{~mm}, h=2 \mathrm{~mm}$, Matl ('g' - global bending, 'p'- progressive buckling).

Figure 12 Counter-intuitive response for circular tubes with $D=50.2 \mathrm{~mm}, h=2.19 \mathrm{~mm}$ and made of material Mat4 subjected to an impact with $V_{0}=10.4 \mathrm{~m} / \mathrm{s}$ and $G=209 \mathrm{~kg}$. (a)

Numerical finite-element predictions for the buckling shapes; (b) Duration of the compressive phases, $t^{*}$ according to the models' prediction $\left(V_{0}=9.6 \mathrm{~m} / \mathrm{s}\right)$.

Figure 13 Influence of the shell thickness on buckling transition $L=550 \mathrm{~mm}, D=50.8 \mathrm{~mm}$, Mat4. (a) Duration of the compressive phases, $t^{*}$, associated with a global bending and progressive buckling depending on the impact velocity; (b) Velocities $V_{1}$ at the proximal end of the models associated with the two buckling modes; (c) Enlarged region from (b) to show velocities $V_{1, \text { glob }}$ and $V_{1, \text { progr }}$ when the global bending mode is dominant $\left(t_{\text {glob }}^{*}<t_{\text {progr }}^{*}\right)$. 
Figure 14

Figure 15

Figure 16

Figure 17

Figure 18

Figure A1.1

Figure A1.2

Figure A1.3

Figure A2.1

Figure A2.2

Figure A2.3
Influence of material strain hardening on the buckling transition; $L=550 \mathrm{~mm}, D=50.8$ $\mathrm{mm}, h=2 \mathrm{~mm}$. (a) Duration of the compressive phases, $t^{*}$, associated with a global bending and progressive buckling depending on the impact velocity; (b) Velocities $V_{1}$ at the proximal end of the models associated with the two buckling modes.

Influence of the material yield stress on buckling transition; $L=550 \mathrm{~mm}, D=50.8$ $\mathrm{mm}, h=2 \mathrm{~mm}$. (a) Duration of the compressive phases, $t^{*}$, associated with a global bending and progressive buckling depending on the impact velocity; (b) Velocities $V_{1}$ at the proximal end of the models associated with the two buckling modes; (c) Characteristic angles for the global bending and progressive buckling at the end of the compression phases $-\varphi\left(t^{*}\right)$ and $\psi\left(t^{*}\right)$, respectively.

Influence of the striking mass on buckling transition of shells made from material Mat4; $D=50.2 \mathrm{~mm}, h=2.19 \mathrm{~mm}, L=500 \mathrm{~mm}, V_{0}=10.4 \mathrm{~m} / \mathrm{s}$. (a) Final buckling shapes; (b) Development of the buckling shape for an impact with $G=500 \mathrm{~kg}$ and $V_{0}=10.4 \mathrm{~m} / \mathrm{s}$; (c) Development of a local fold, $\Psi$, and global bending, $\Phi:-G=500$ $\mathrm{kg} ;$----- $G=209 \mathrm{~kg} ; L=500 \mathrm{~mm}, V_{0}=10.4 \mathrm{~m} / \mathrm{s}$.

Impact velocity ranges for the different modes of collapse of shells with $D=50.8 \mathrm{~mm}$, $h=2 \mathrm{~mm}$ made from Mat1 depending on the loading conditions. (a) Constant impact energy, $T_{0}=5 \mathrm{~kJ}, L_{1}=550 \mathrm{~mm}, L_{2}=650 \mathrm{~mm}$; (b) Different impact masses: $G_{1}=200$ $\mathrm{kg}, G_{2}=500 \mathrm{~kg} ; L=650 \mathrm{~mm}$.

Critical shell lengths for dynamic buckling transition; $D=50.8 \mathrm{~mm}, h=2 \mathrm{~mm} ; \diamond$ calculated points according to the analytical models, $x$ - points resulting from the finite-element numerical simulations, $T_{0}=5 \mathrm{~kJ}$ [9]. (a) Mat1, ' 1 ' - according to condition (34), $T_{0}=5 \mathrm{~kJ}$, ' 2 ' - according to Eq. (42), $T_{0}=5 \mathrm{~kJ}$; ' 3 ' - according to Eq. (42), $G=500 \mathrm{~kg}$. (b) Variation of the shell lengths at buckling transition depending on the impact velocity and material strain hardening modulus, $T_{0}=5 \mathrm{~kJ}$.

Duration of the compression phases depending on the initial imperfections for two tube lengths; $D=50.8 \mathrm{~mm}, h=2 \mathrm{~mm}$, Mat1. (a) $L=450 \mathrm{~mm}$; (b) $L=750 \mathrm{~mm}$.

Variation of the characteristic angles $\varphi\left(t^{*}\right)$ and $\psi\left(t^{*}\right)$ depending on the impact velocity and initial imperfections.(a) Angle $\varphi\left(t^{*}\right)$ for $L=750 \mathrm{~mm}$ and two values of the initial imperfections; (b) $\varphi_{\max }\left(t^{*}\right)$ depending on the initial imperfections; (c) Angle $\psi\left(t^{*}\right)$ for $\mathrm{L}=750 \mathrm{~mm}$ and two values of the initial imperfections.

Imperfection sensitivity of the velocity of the striker at $t=t^{*}$.

Buckling shapes of shells (a) Numerical simulation; $D=50.2 \mathrm{~mm}, h=2.19 \mathrm{~mm}, L=$ $381 \mathrm{~mm}, \sigma^{0}=250 \mathrm{MPa} ; V_{0}=5.8 \mathrm{~m} / \mathrm{s}$; (b) Experimental result [9]; $D=50.28 \mathrm{~m}, h=$ $2 \mathrm{~mm}, L=500 \mathrm{~mm}$; the stress-strain curve is given in Fig. A2.2; $V_{0}=9 \mathrm{~m} / \mathrm{s}, G=$ $200 \mathrm{~kg}[9]$.

The actual stress-strain curve for the experimental results shown in Fig. 1(c) [9] together with the model materials Mat1 and Mat2.

Variation of the vertical velocity at the proximal end of a tube at the initiation of each subsequent fold formed progressively, $T_{p, f}=1000 \mathrm{~J}, V_{s f}=\sqrt{2 T_{p, f} / G}$. (a) Constant impact energy, $T_{0}=5 \mathrm{~kJ}$; (b) Constant striking mass. 
Table 1: Material properties

\begin{tabular}{lcccc}
\hline & Mat1 & Mat2 & Mat3 & Mat4 \\
\hline$\sigma_{0}(\mathrm{MPa})$ & 175 & 175 & 105 & 260 \\
\hline$E_{h}(\mathrm{MPa})$ & 300 & 500 & 500 & 446 \\
\hline
\end{tabular}




\section{Appendix 1 - Imperfection sensitivity analysis}

An imperfection sensitivity analysis is presented for the models, which describe the axial compression phases. The initial imperfections for both models in Fig. 6 are governed by the global buckling mode. In order to compare the amplitudes' growth starting from the same disturbances, the initial angles of rotation are always equal: $\varphi_{0}=\psi_{0}$. This choice can be also allied with the physical problem where a small initial curvature of long tubes is within the manufacturing tolerance, which can be characterised e.g., by an initial angle. An independent variation of the imperfections for the two modes can lead to arbitrary results.

The present study suggests that the relative variation of the duration of the axial compression phases of the two principal buckling modes, which exhibit imperfection sensitivity, is the major factor that controls the dynamic buckling transition of a tube. Together with this, the lateral/transverse displacements at the initiation of the bending phases (large deformation or post-buckling phases), which are influenced by the compression phase, give an indication of the rationality of the models' performance.

The imperfection sensitivity analysis is presented here for tubes made from material Mat1 with $D$ $=50.8 \mathrm{~mm}, h=2 \mathrm{~mm}$ and for two tube lengths, $L=450 \mathrm{~mm}$ and $L=750 \mathrm{~mm}$. The imperfections in terms of initial angles are varied within the interval $\left(\varphi_{0}, \psi_{0}\right) \in[0.00075,0.003]$. Figure A1.1 shows the variation of the duration of the compression phases, $t^{*}$, for the two buckling modes of tubes having two different lengths together with the corresponding velocities for the initiation of a buckling transition, $V_{0, t}$. One can see that the duration of the compression phase for the global bending mode is very sensitive to the initial imperfections, while the compression phase for the progressive mode is only slightly affected. This observation is consistent with the buckling phenomena in long tubes having given cross-sectional characteristics; shorter compression phases and higher impact velocities for the initiation of progressive buckling are predicted for the larger initial imperfections.

The rotational angles of the links of the models in Figs. 6(a) and 6(a), representing global and progressive buckling modes, respectively, increase during the axial compression phase and at $t=t^{*}$ reach values, which depend on all the other parameters of the problem. The variation of the global bending mode characteristic, $\varphi\left(t^{*}\right)$, is plotted in Fig. A1.2(a) where this angle increases with the impact velocity but decreases for impact velocities $V_{0} \geq V_{0, t}$, which initiate progressive buckling. The maximum values of $\varphi\left(t^{*}\right)$ are shown in Fig. A1.2(b) for the maximum values of the initial imperfections used in the analysis. It is noted that a fourfold increase of the initial imperfection magnitudes cause about a $16 \%$ increase of $\varphi\left(t^{*}\right)$. By way of contrast, the rotational angle $\psi\left(t^{*}\right)$ continues to increase with the impact velocity until the conditions for a global bending intervene; this angle reaches a maximum value at $V_{0}=V_{0, t}$ and remains constant for $V_{0} \geq V_{0, t}$ (Fig. 
A1.2(c)). For the particular cross-sectional characteristics studied, $\psi_{\max }\left(t^{*}\right)$ is virtually independent of the initial imperfections, but varies slightly with the tube length. $\psi_{\max }\left(t^{*}\right)=0.1219$ for $L=450 \mathrm{~mm}$ and $\psi_{\max }\left(t^{*}\right)=0.1187$ for $L=750 \mathrm{~mm} . \psi_{\max }\left(t^{*}\right)$ determines $w_{\max }\left(t^{*}\right)_{\mid L=450 \mathrm{~mm}}=0.585 h$ and $w_{\max }\left(t^{*}\right)_{\mid L=750 \mathrm{~mm}}=0.57 \mathrm{~h}$ at the end of the corresponding axial compression phases, which justify the assumption for small transverse displacements during compression.

As a consequence of the variation of the compression phase, the velocity of the striker at $t=t^{*}$ also can exhibit sensitivity to the initial imperfections. This analysis is shown in Fig. A1.3 where it is evident that only marginal differences occur for material Mat1 with a low hardening modulus.

Initial imperfections $\varphi_{0}=\psi_{0}=0.0015$ are used in the present study mainly to facilitate a comparison (where it is possible) with the numerical simulations. 


\section{Appendix 2 - Energy dissipated in a single fold, $T_{p, f}$}

The energy absorbed during the development of an axisymmetric fold can be calculated as [3]

$$
T_{p, f}=2 \pi \sigma^{0} h\left\{h(\pi R+\tilde{l}) / \sqrt{3}+\tilde{l}^{2}\right\}
$$

where $\tilde{l}=(\pi R h / \sqrt{3})^{1 / 2}$ [2]. Equation (A2.1) predicts the energy absorption for materials having a negligible strain hardening since a perfectly plastic formulation has been used and is recommended for circular tubes with ratios $D / h \leq 40-45$.

It is shown, however [18], that circular shells made from materials, which exhibit strain hardening properties, undergo axial compression (an overall reduction of the shell length) during the entire response, i.e. some energy is also absorbed in axial compression during the development of each fold. Therefore, Eq. (A2) largely underestimates the energy absorption, particularly for thicker tubes similar to those with $D / h$ ratios within the approximate range $23-25$ studied in the present paper.

The buckling shapes with axisymmetric folds obtained numerically and experimentally shown in Fig. A2.1 suggest that Eq. (A2.1) can be potentially used to estimate the number of folds as $n \approx T_{0} / T_{p, f}$. The tubes from the numerical simulations shown in Fig. A2.1(a) have $D=50.2 \mathrm{~mm}$ and $h=2.19 \mathrm{~mm}$ (Mat4), while the tube from the experimental programme shown in Fig. A2.1(b) is thinner having $D=50.8 \mathrm{~mm}$ and $h=2 \mathrm{~mm}$ (the experimental stress-strain curve is shown in Fig. A2.2). The dissipated energy due to the plastic deformations $T_{p, f}=817 \mathrm{~J}$ is obtained for the tubes in Fig. A2.1(a) when using $\sigma^{0}=280 \mathrm{MPa}$, which suggests that four folds can be expected for an impact energy of $3.515 \mathrm{~kJ}(G=209 \mathrm{~kg})$ and ten folds for an impact with $T_{0}=8.41 \mathrm{~kJ}(\mathrm{G}=500 \mathrm{~kg})$, while only two and a half folds and seven folds, respectively, are obtained in the numerical simulations. This observation is also evident from the experimental results, e.g. tube in Fig. A2.1(b), which buckles progressively with eight folds, while the plastic energy according to Eq. (A2.1) is $543 \mathrm{~J}$, which predicts 15 folds for $T_{0}=8.1 \mathrm{~kJ}$. Therefore, the energy $T_{p, f}$ according to Eq. (A2.1) would greatly overestimate the critical impact velocity for buckling transition if used in Eq. (42).

In order to use the transition criterion given by Eq. (42) it is suggested that experimental or numerical data could be used to estimate $T_{p, f}$ when relatively small $D / h$ ratios and materials with considerable strain hardening properties are analysed. Since the model materials Mat1 and Mat2 are close to the actual material for the tube in Fig. A2.1(b), $T_{p, f} \approx T_{0} / n=1000 \mathrm{~J}$ is used to construct Figs. 17 and 18 in the present paper.

Variation of the vertical velocity at the proximal end of a tube at the initiation of each subsequent fold, which forms progressively, is shown in Fig. A2.3(a) and Fig. A2.3(b) for impact loadings with a constant impact energy and with constant masses, respectively. 


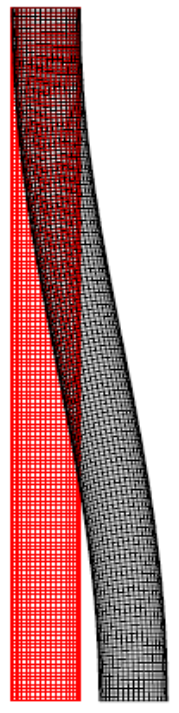

(a)

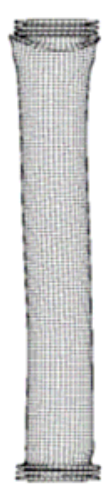

(b)

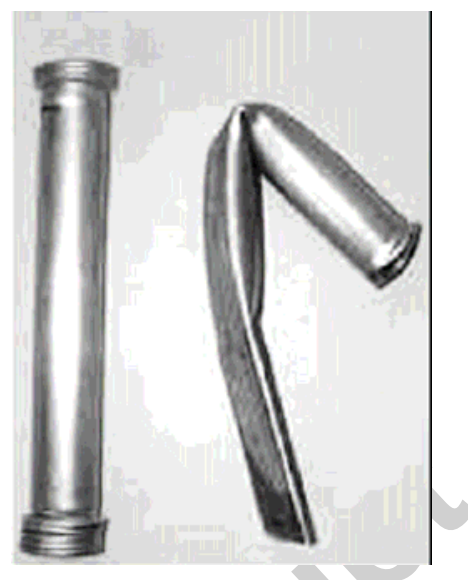

(c)

Figure 1

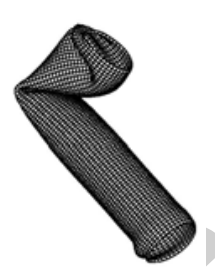

$1.8 \mathrm{~m} / \mathrm{s}$

(a)

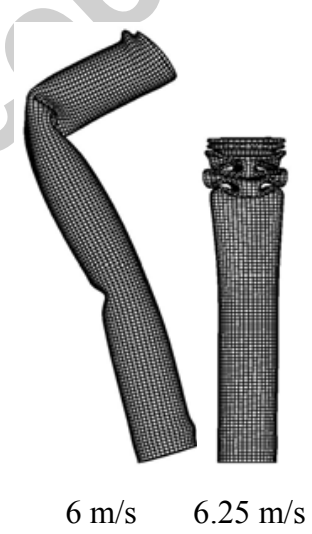

(e)

(f)

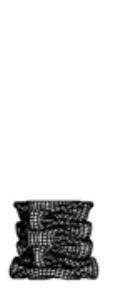

$2.0 \mathrm{~m} / \mathrm{s}$

(b)

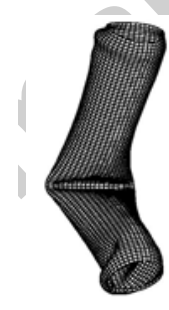

$2.5 \mathrm{~m} / \mathrm{s}$

(c)

$2.8 \mathrm{~m} / \mathrm{s}$

(d)

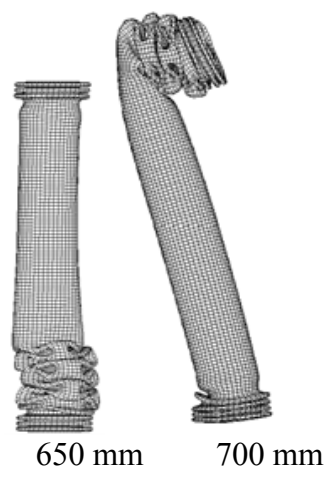

(g)

(h)

Figure 2 


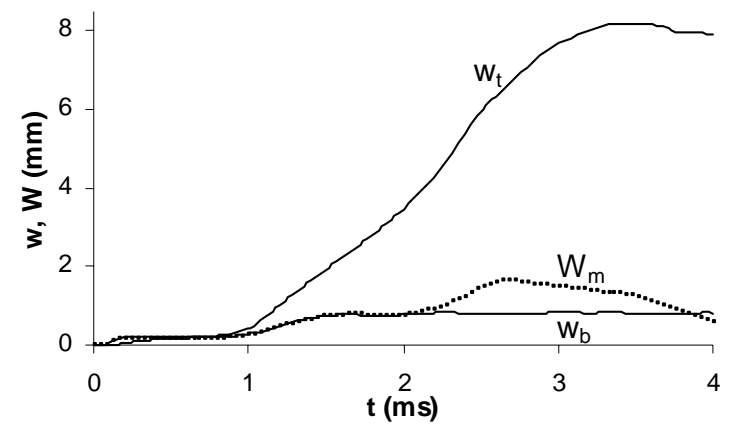

(a)

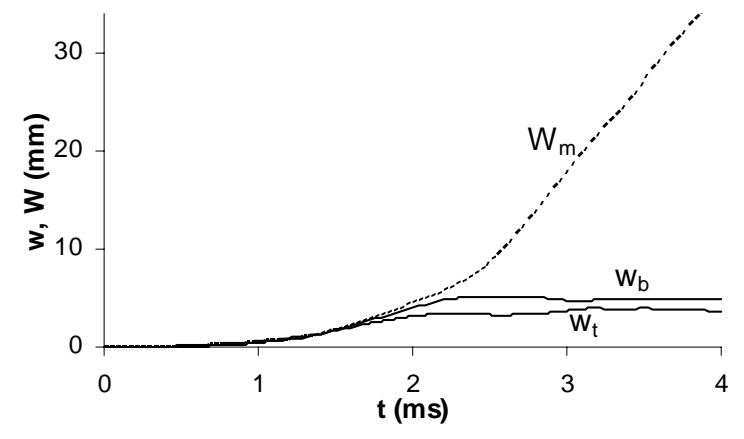

(b)

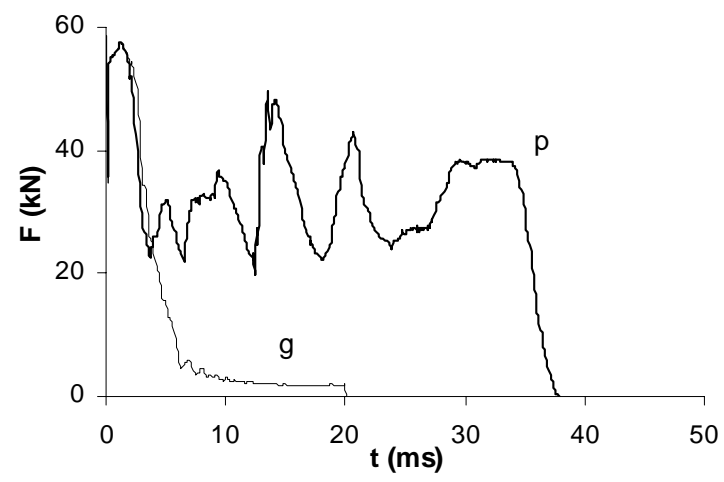

(c)

Figure 3

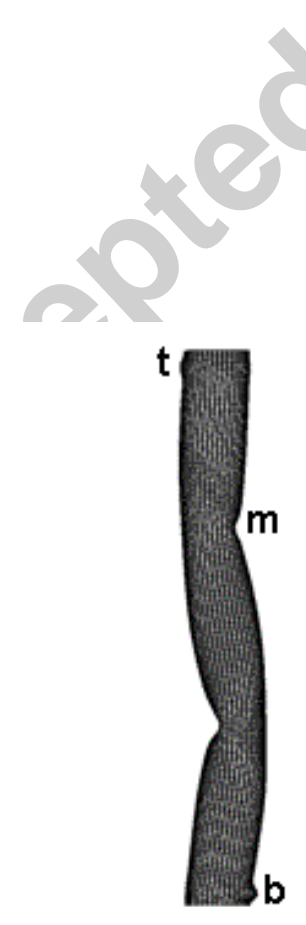

(a)

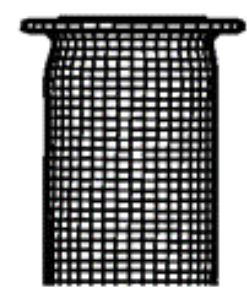

(b)

Figure 4 

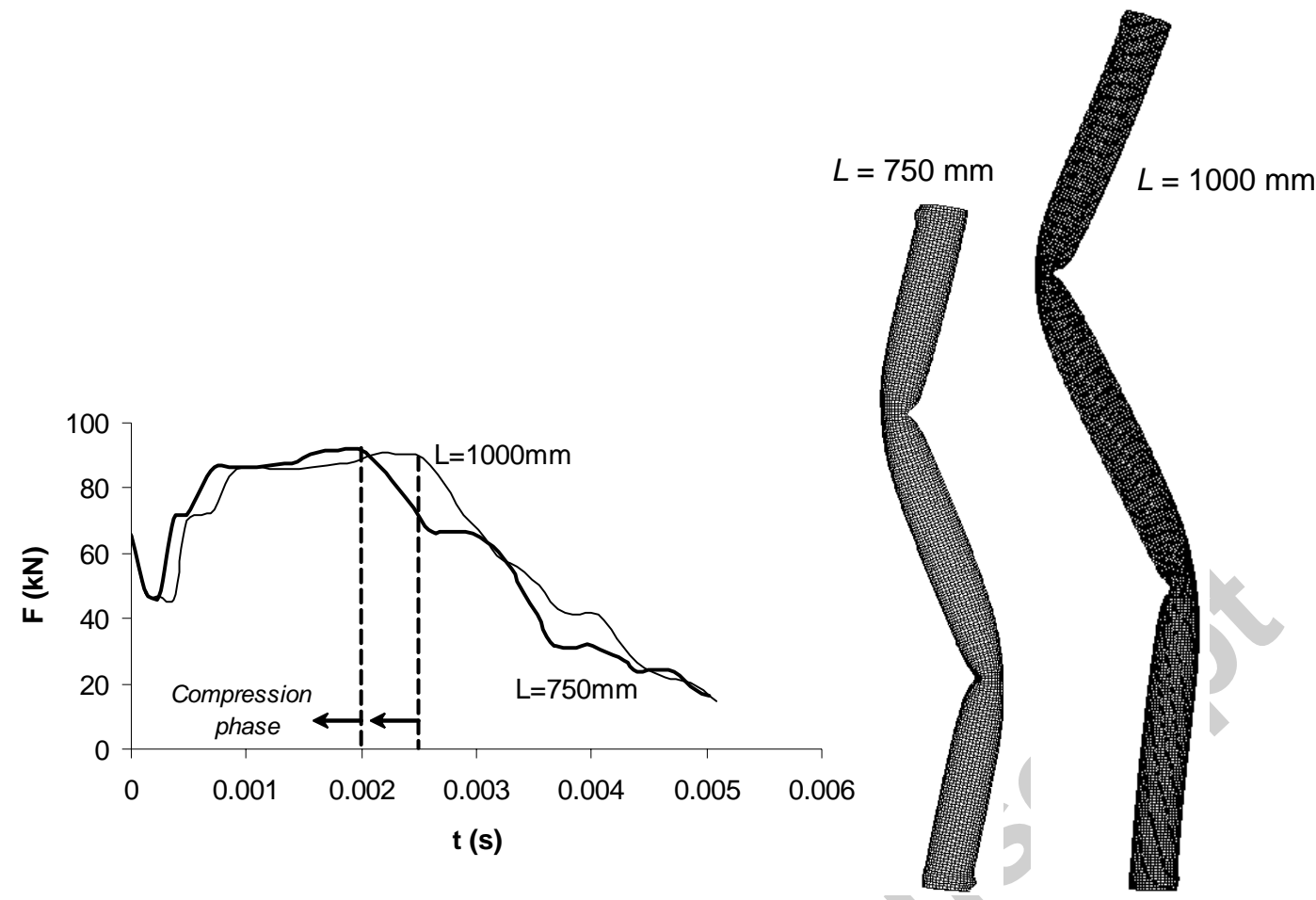

(a)
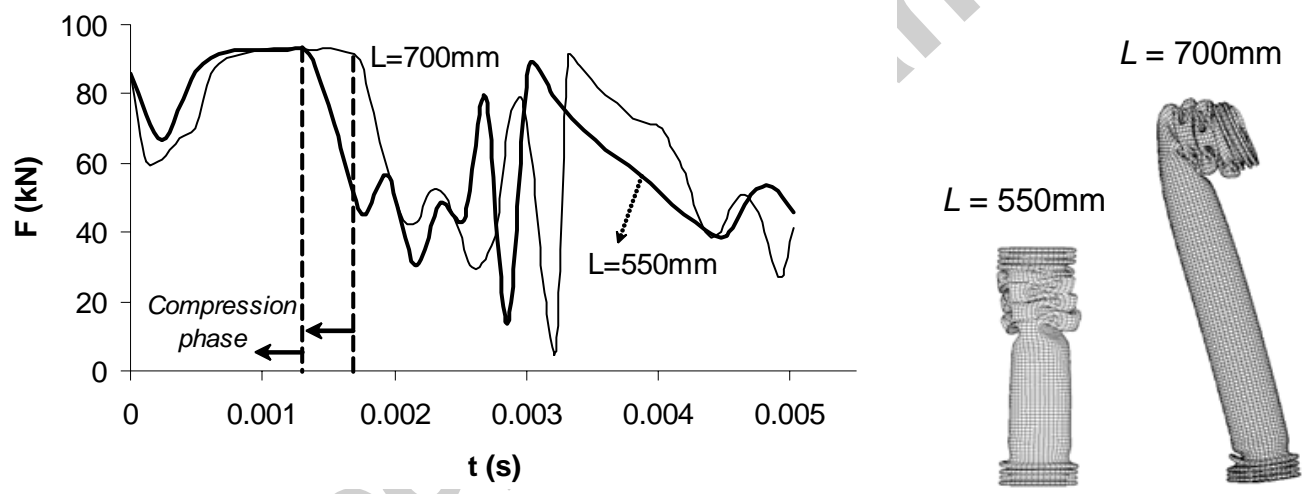

(b)
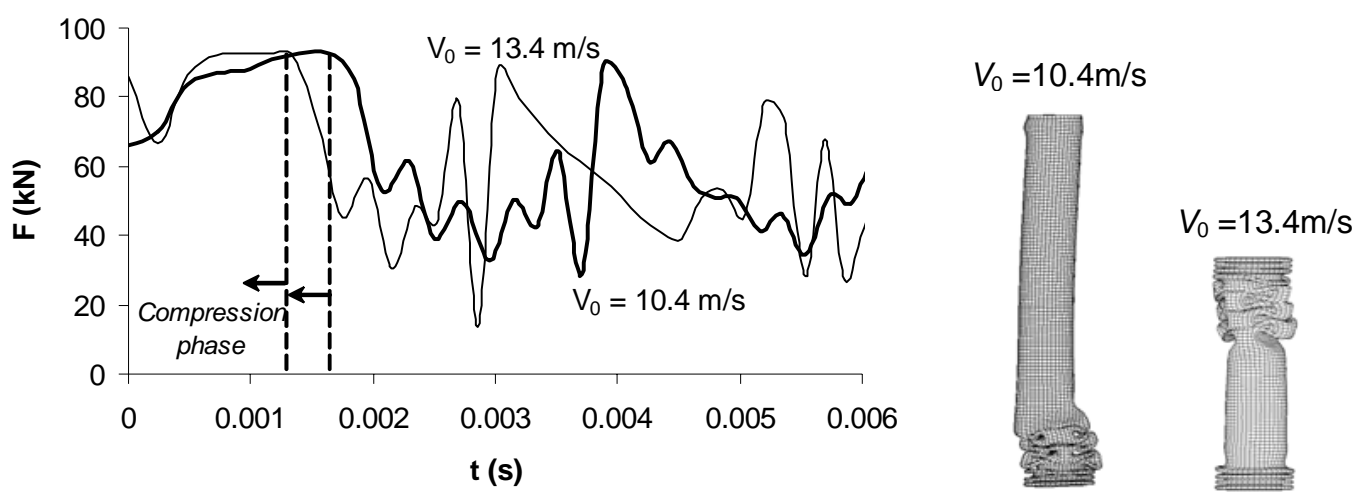

(c)

Figure 5 


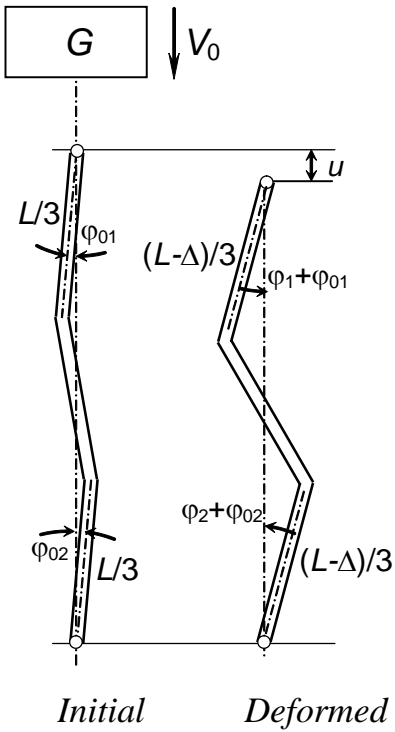

(a)

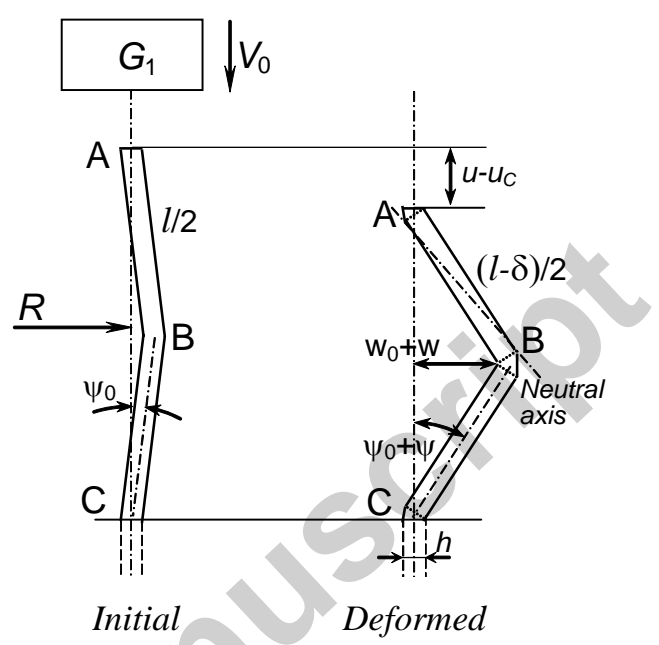

(b)

Figure 6

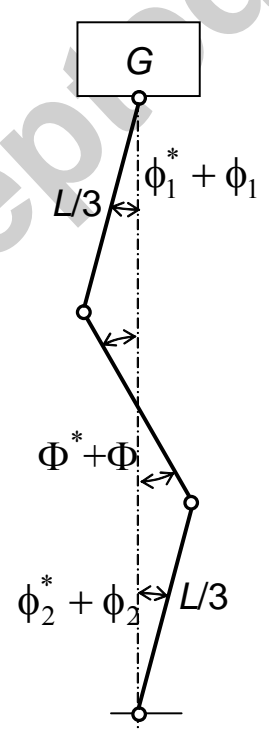

(a)

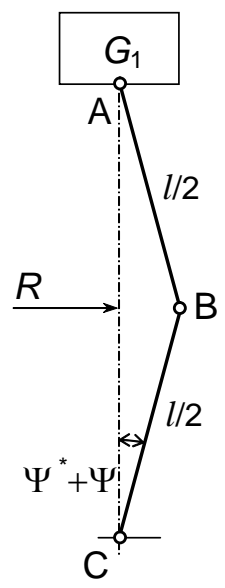

(b)

Figure 7 

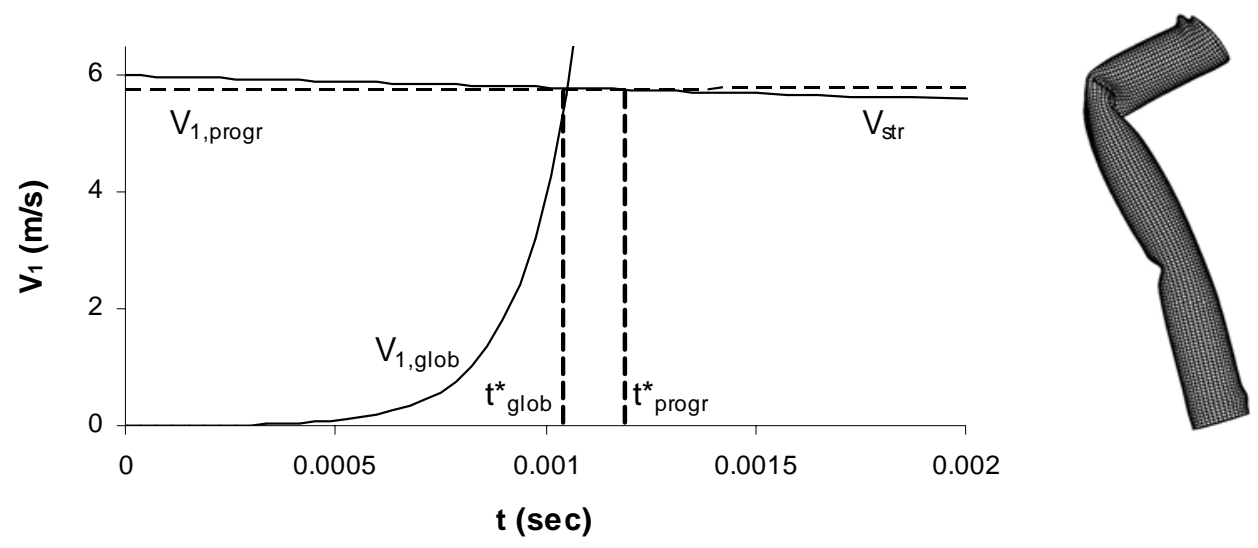

(a)
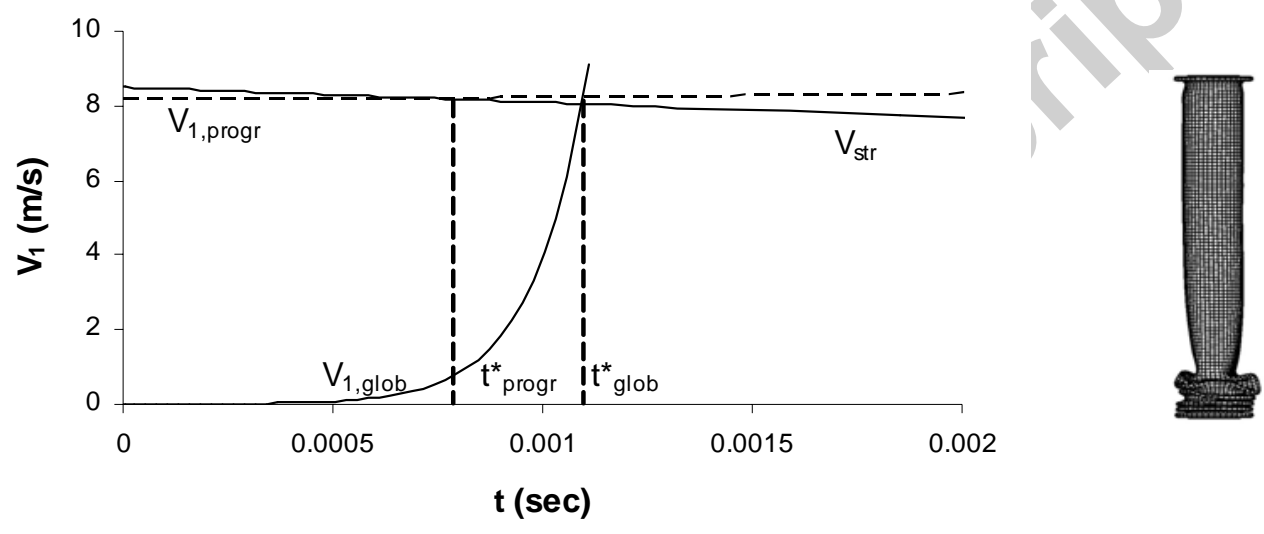

(b)

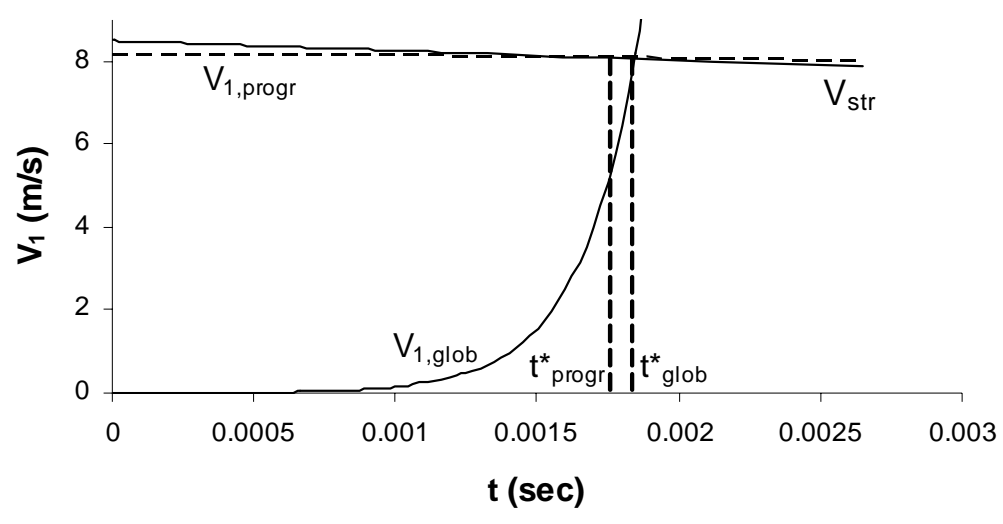

(c)

Figure 8 


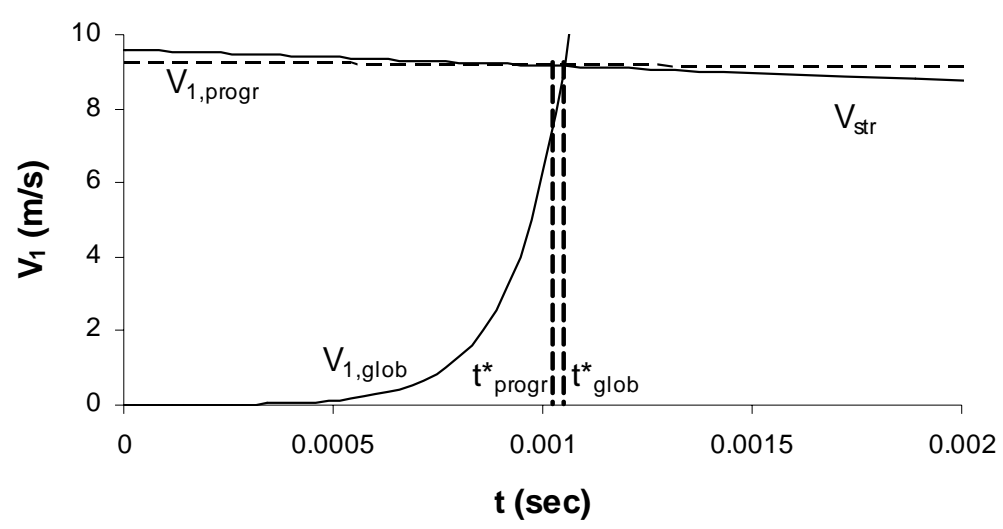

(a)

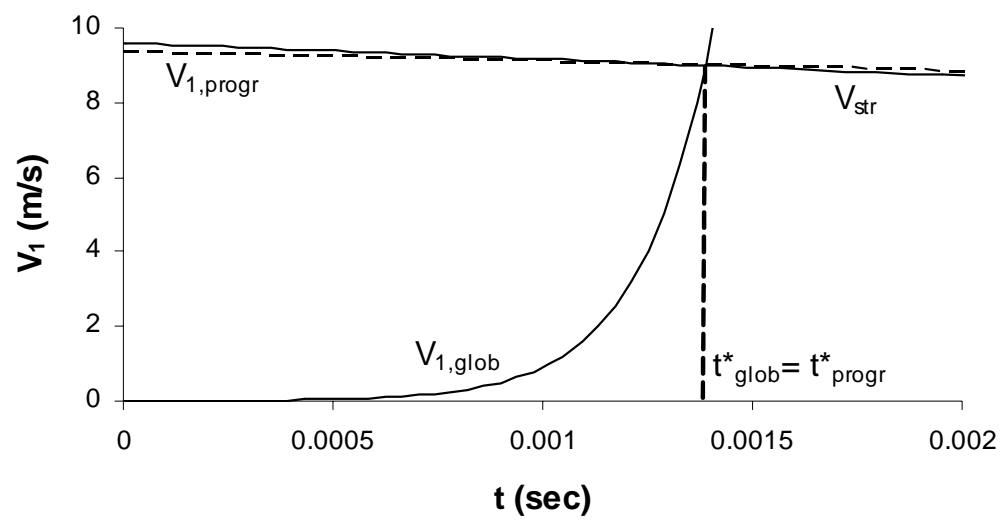

(b)

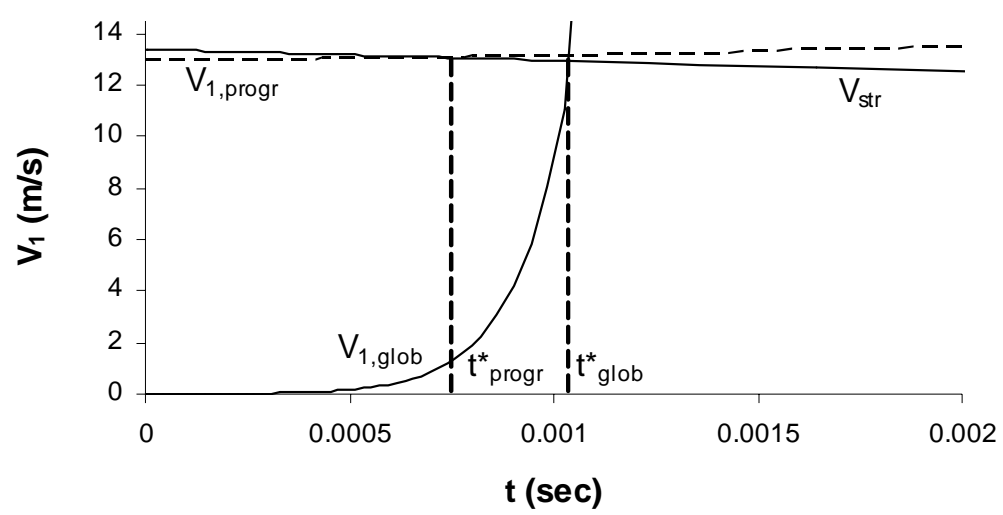

(c)

Figure 9 


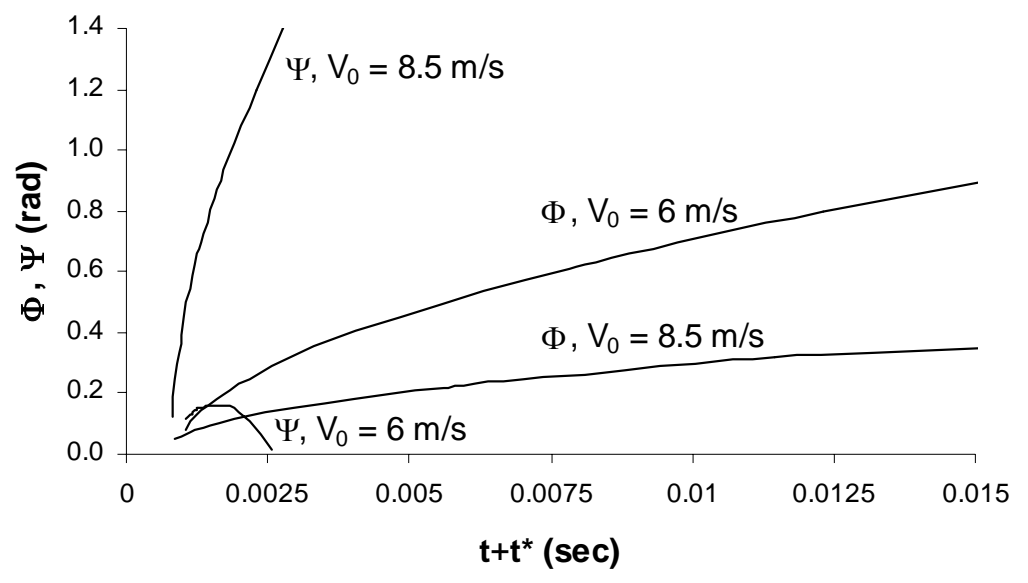

(a)

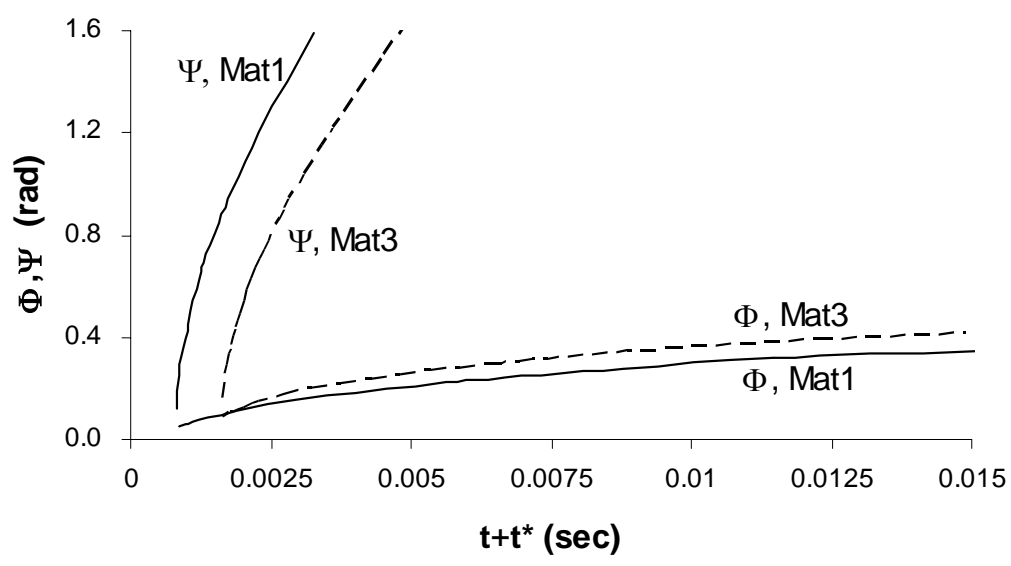

(b)

Figure 10

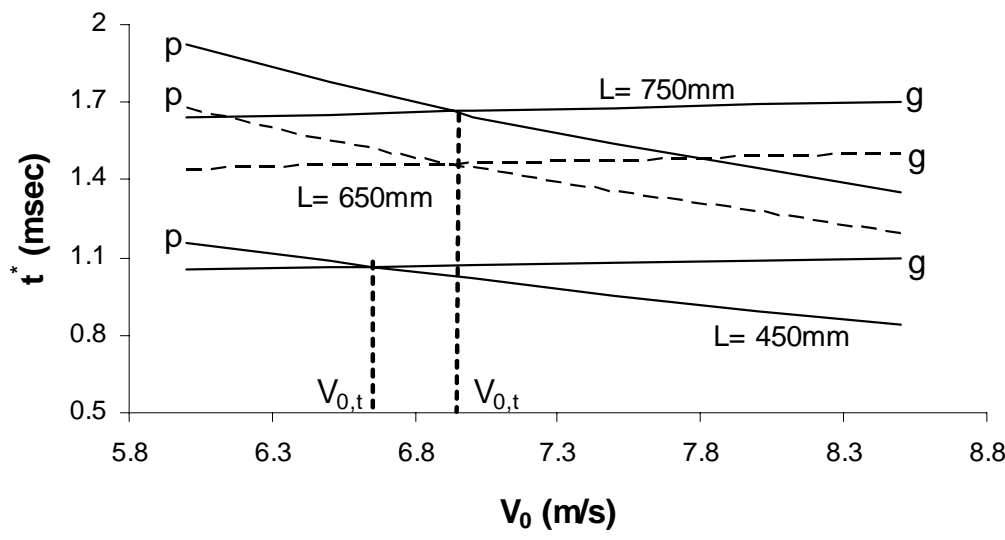

Figure 11 


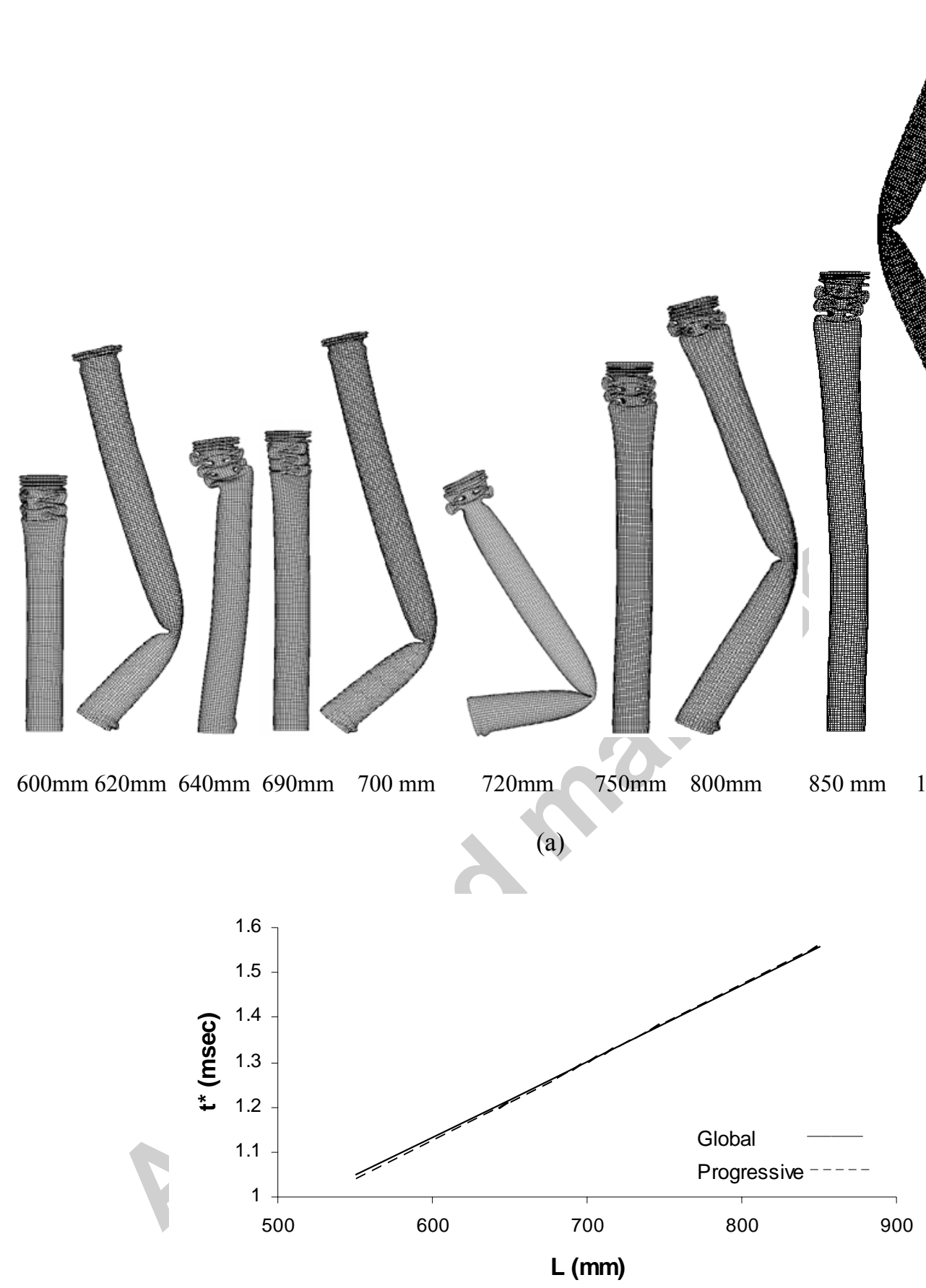

(b)

Figure 12 


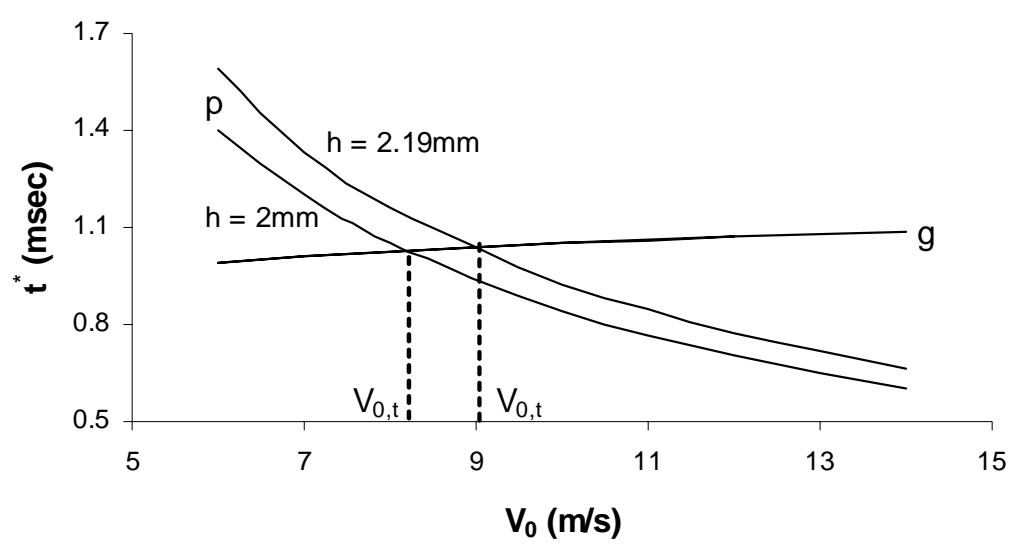

(a)

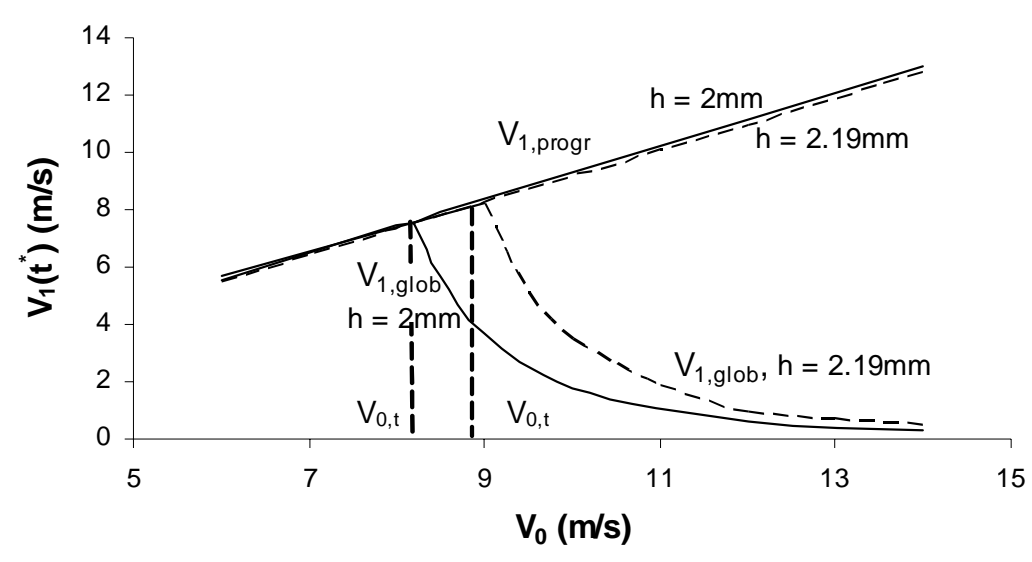

(b)

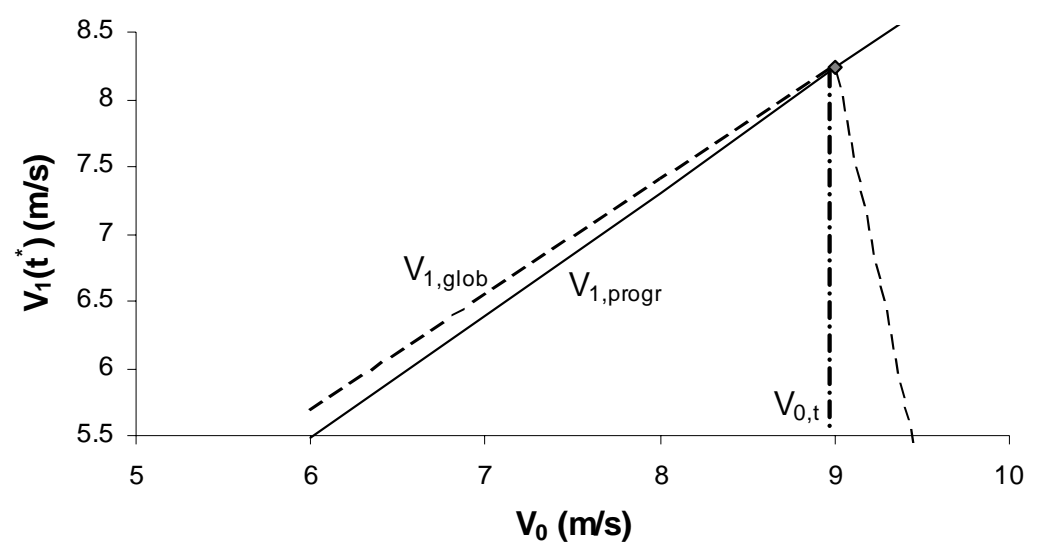

(c)

Figure 13 


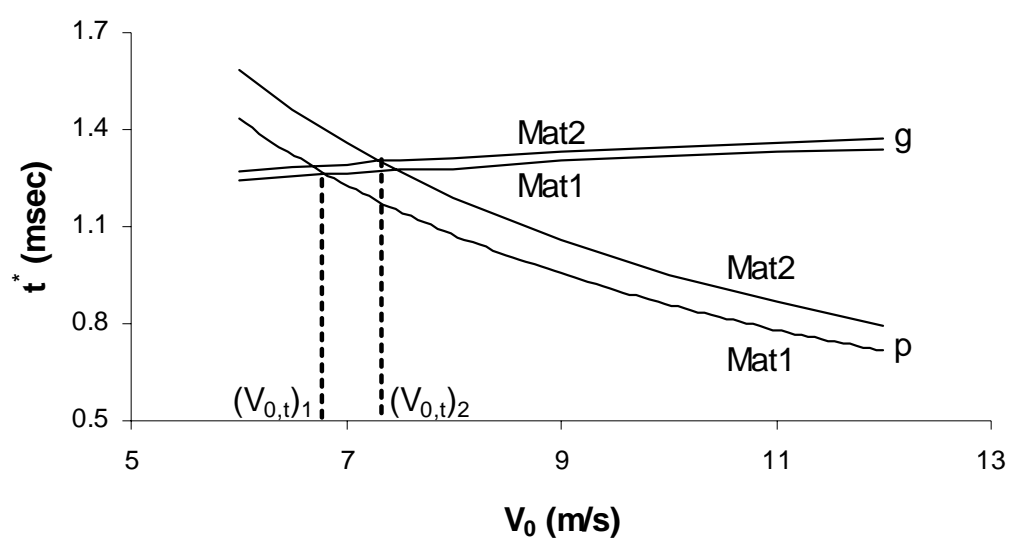

(a)

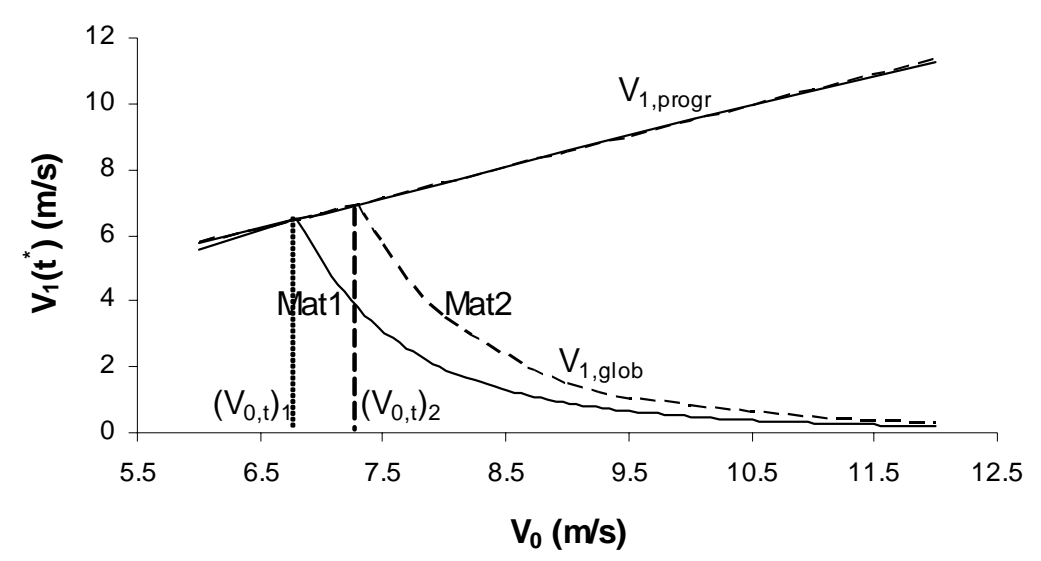

(b)

Figure 14 


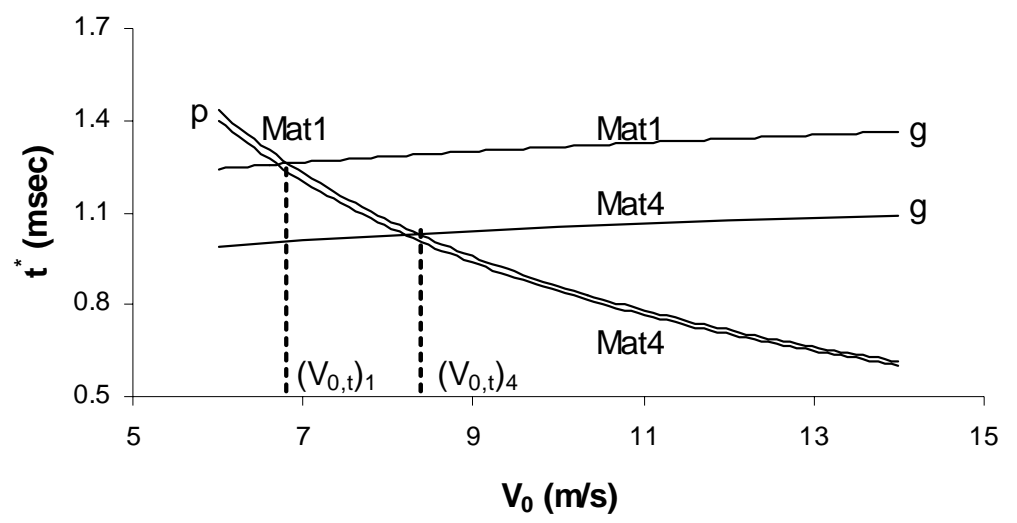

(a)

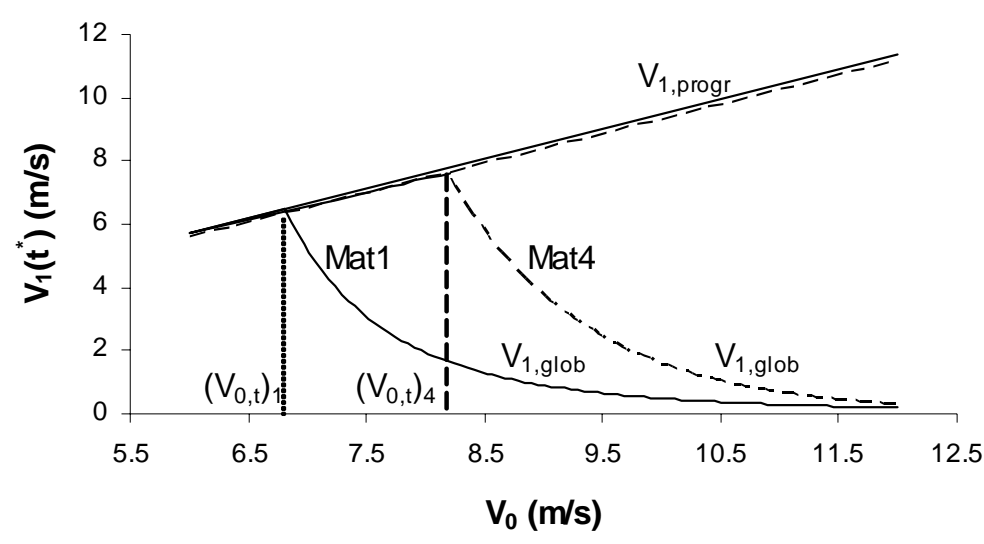

(b)

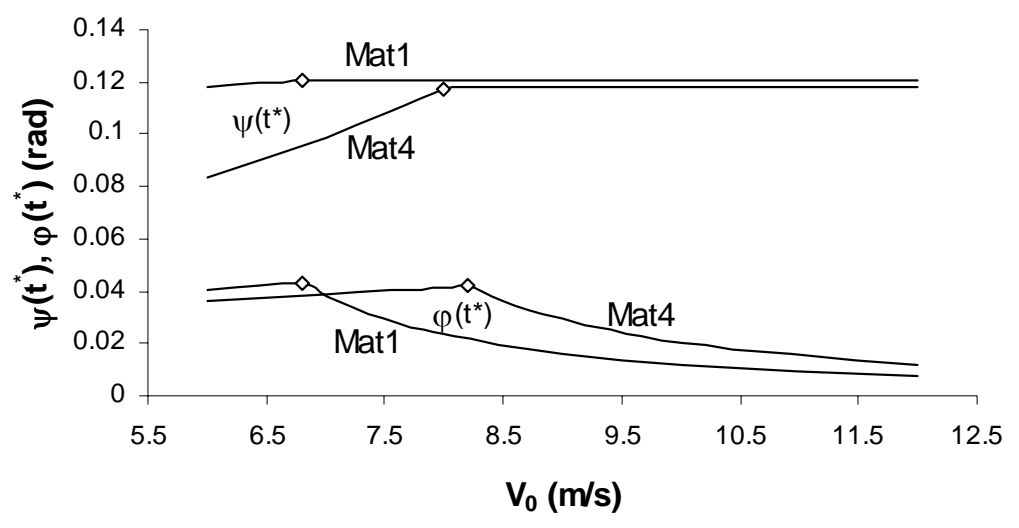

(c)

Figure 15 


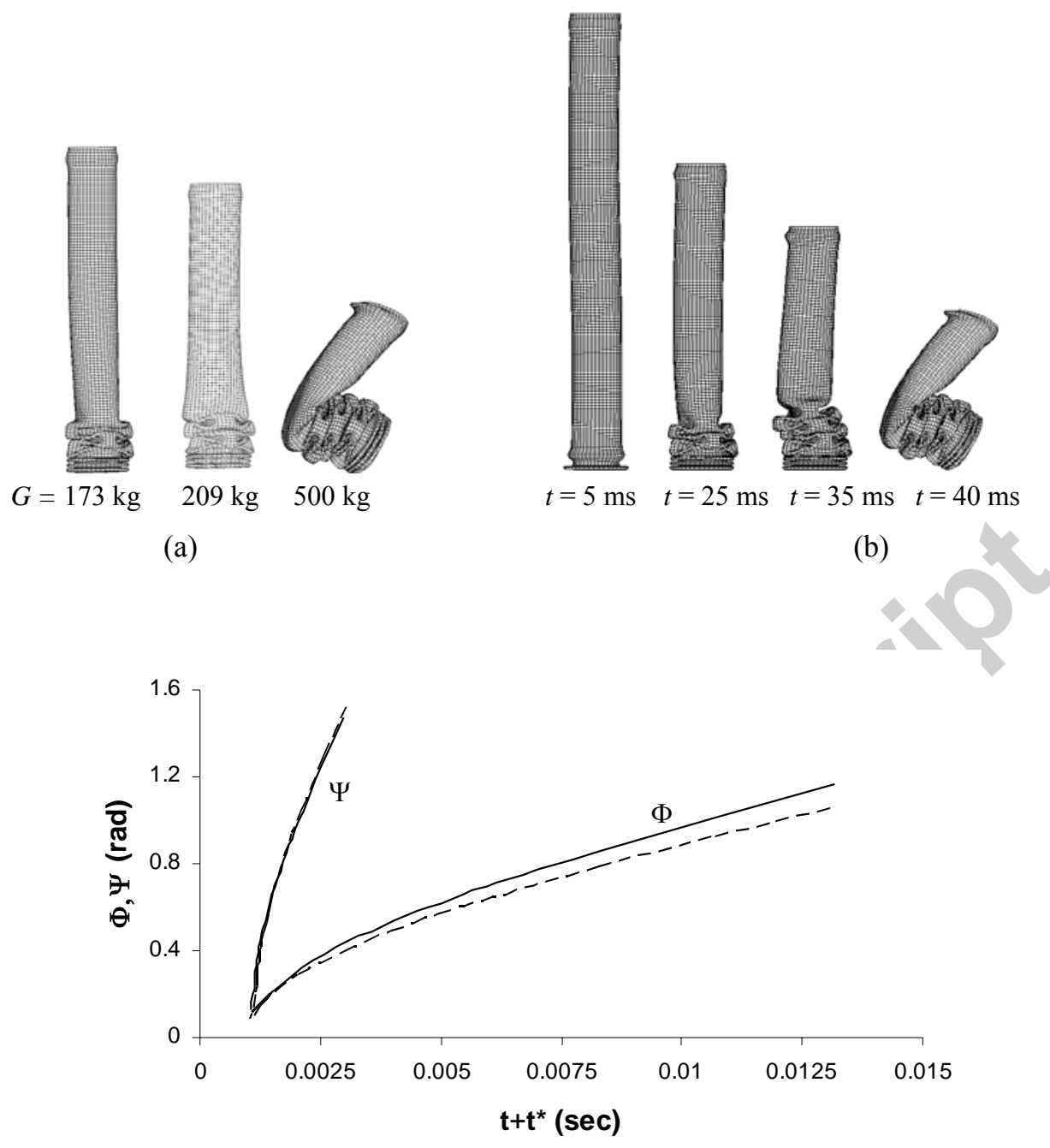

(c)

Figure 16 


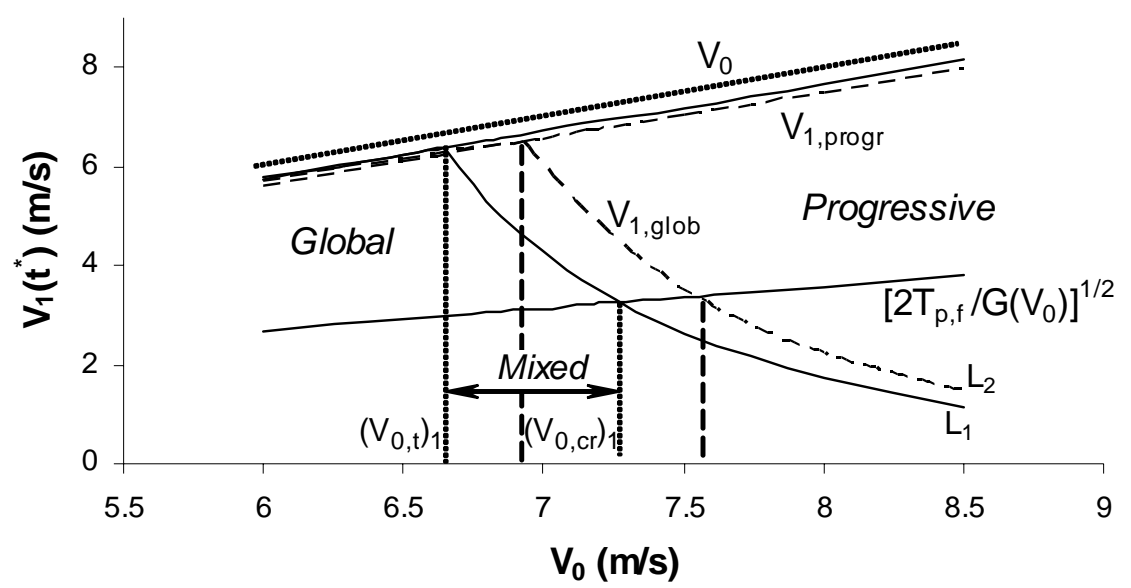

(a)

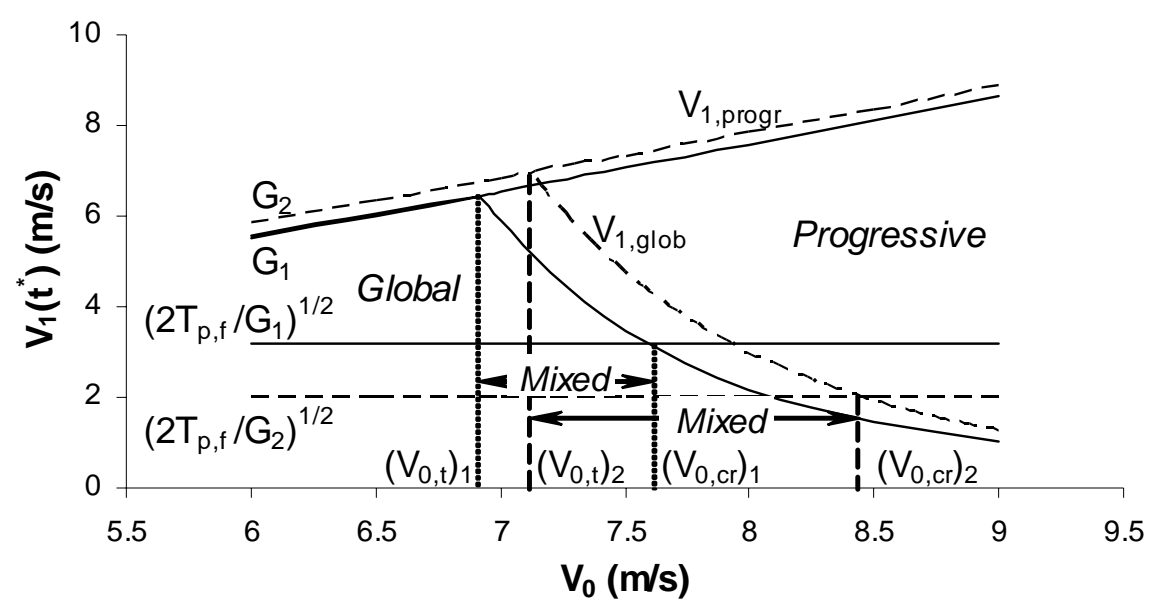

(b)

Figure 17 


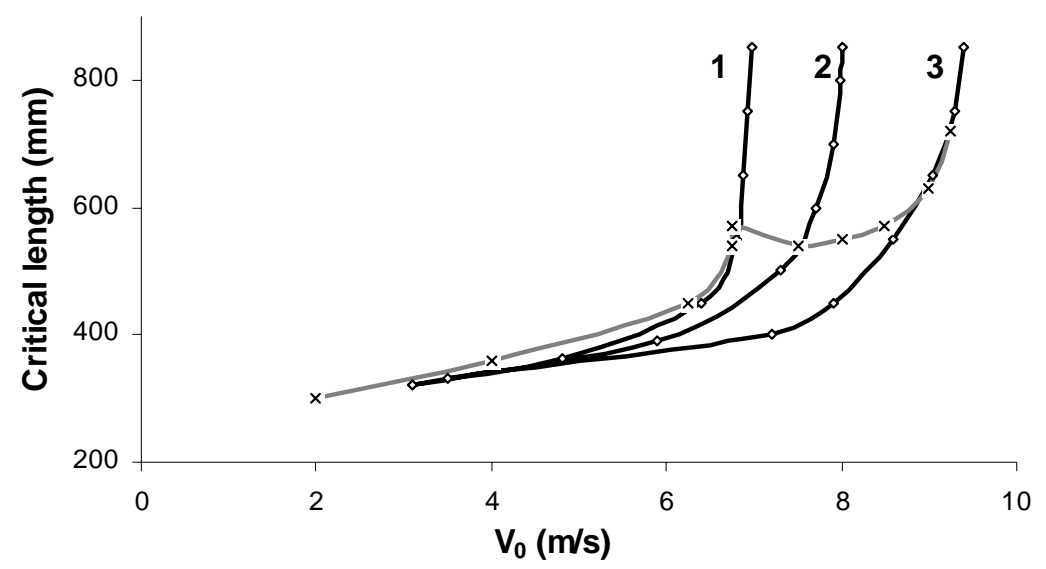

(a)

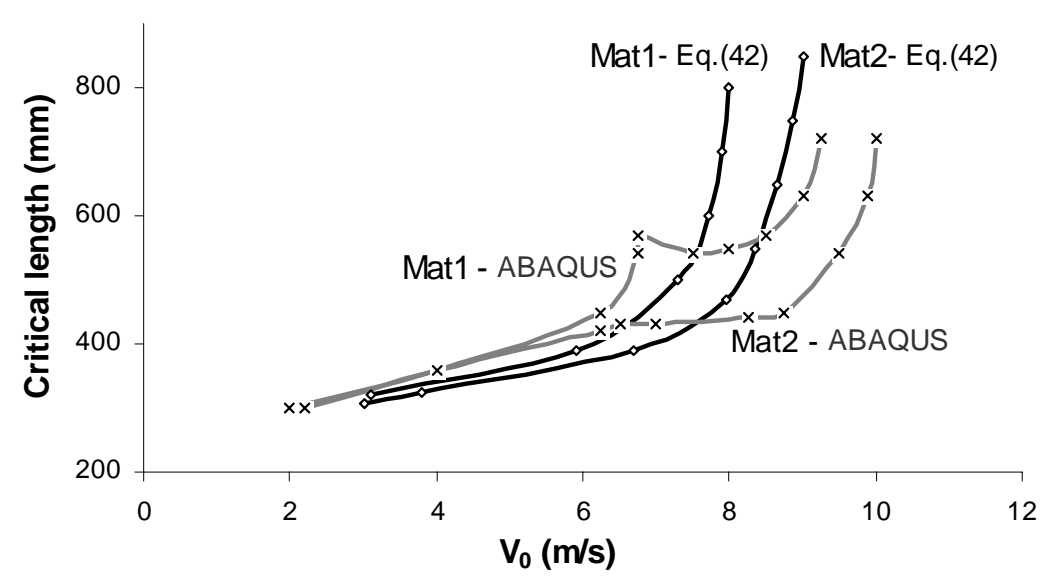

(b)

Figure 18 


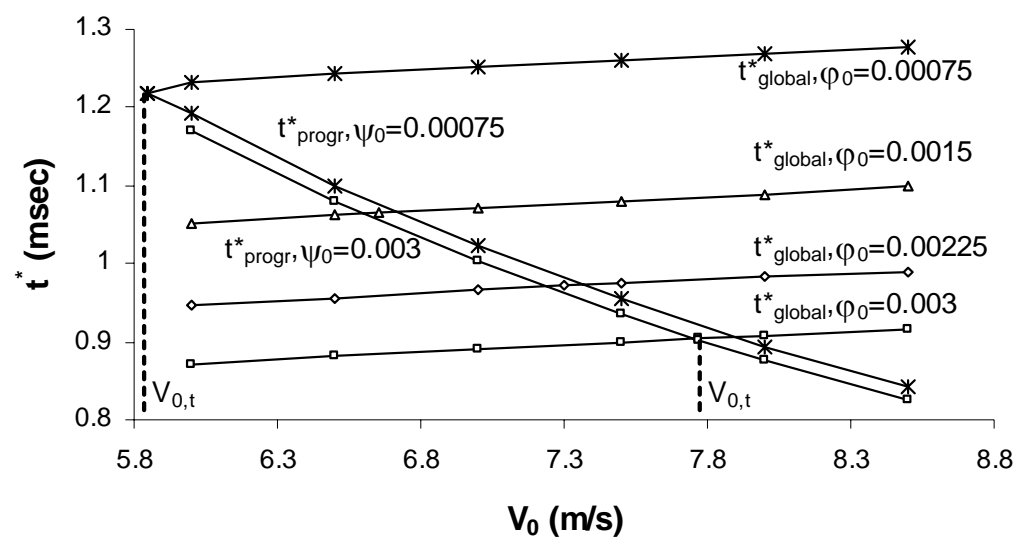

(a)

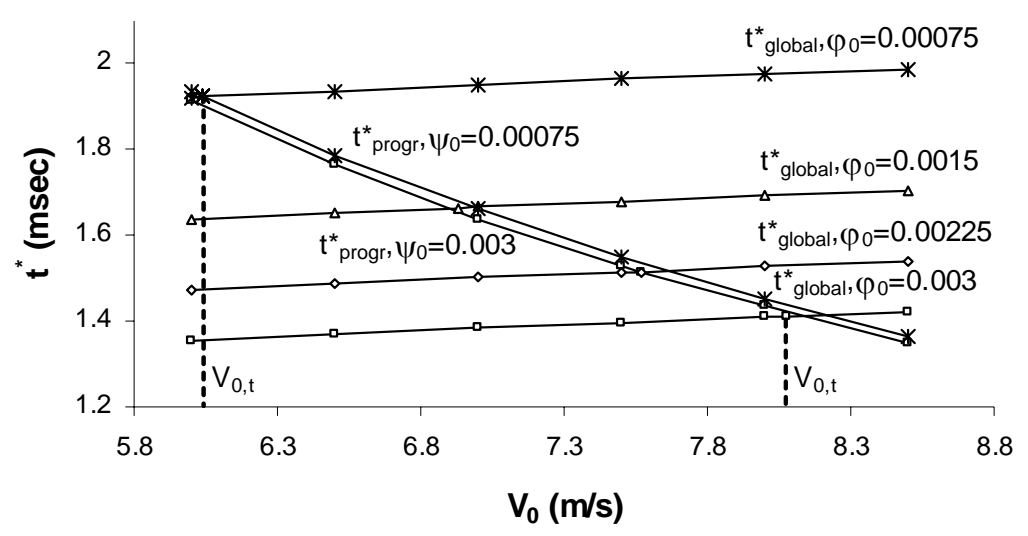

(b)

Figure A1.1 


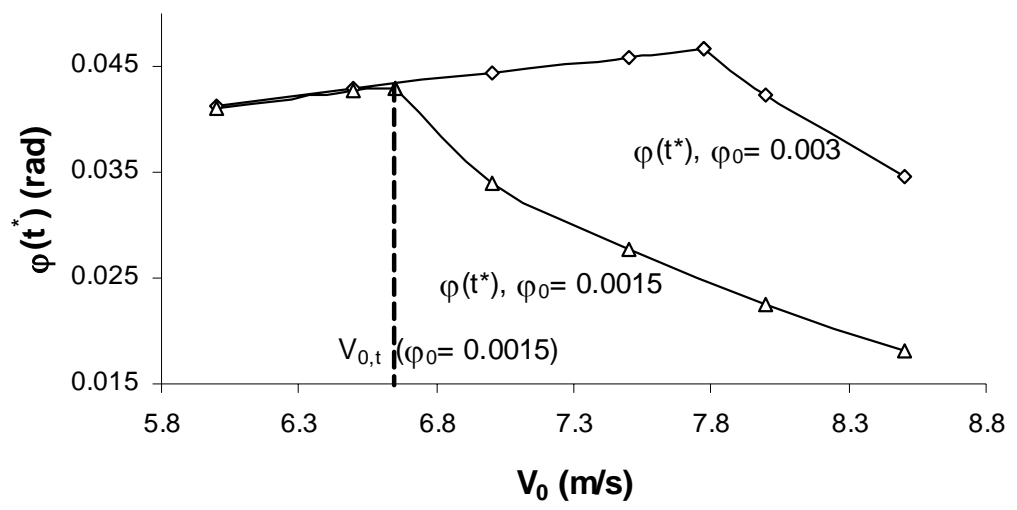

(a)

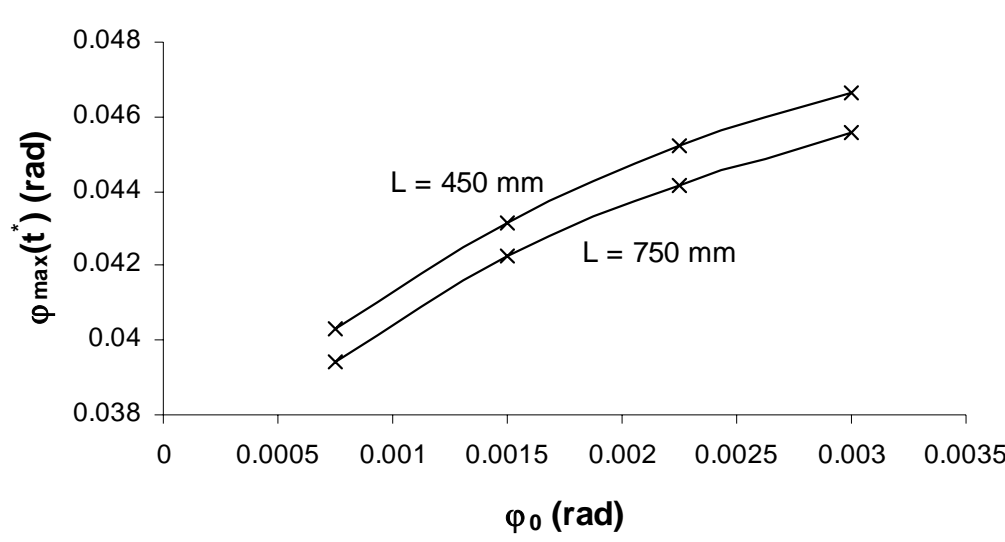

(b)

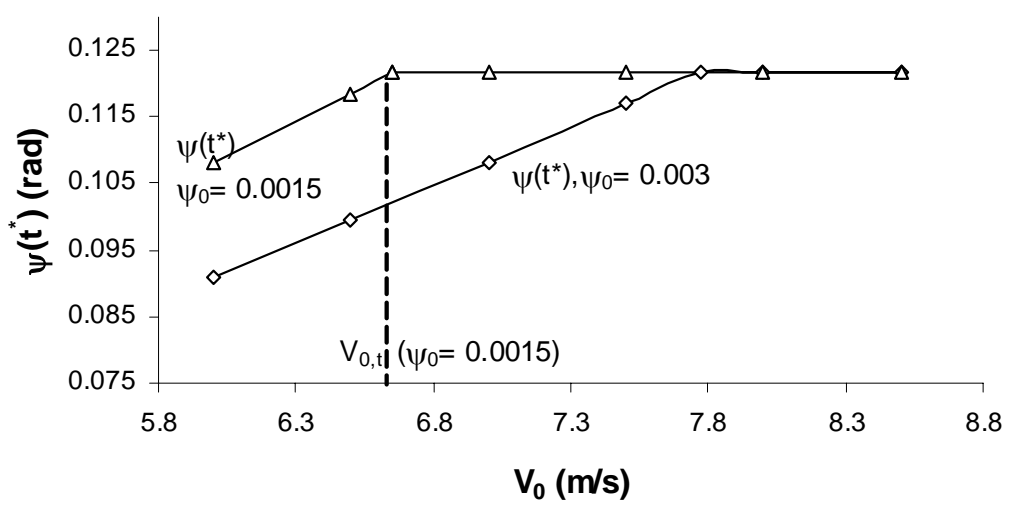

(c)

Figure A1.2 


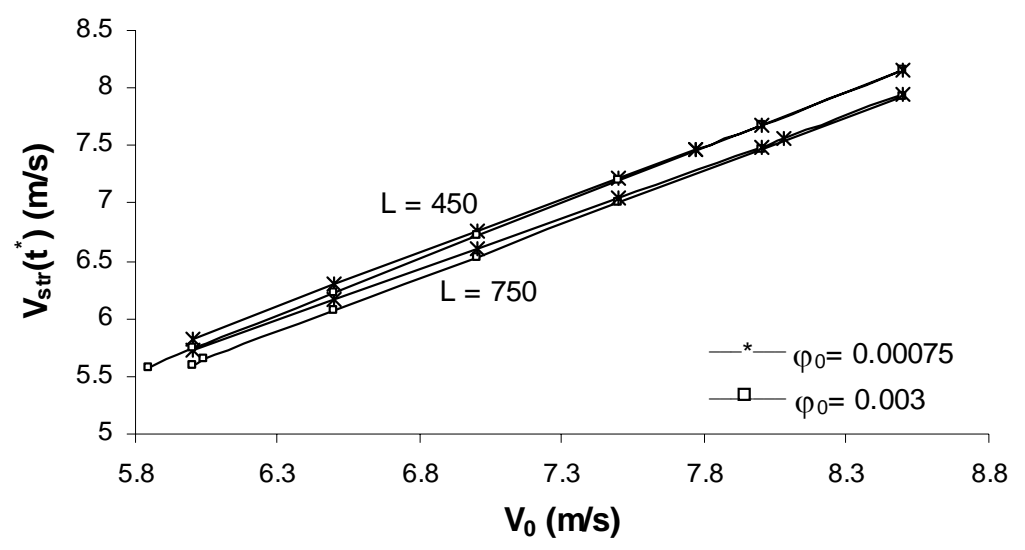

Figure A1.3
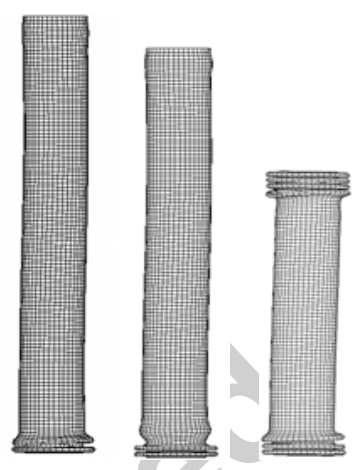

$173 \mathrm{~kg} 209 \mathrm{~kg} 500 \mathrm{~kg}$

(a)

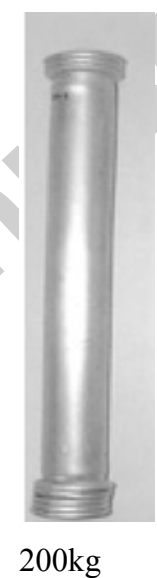

(b)

Figure A2.1

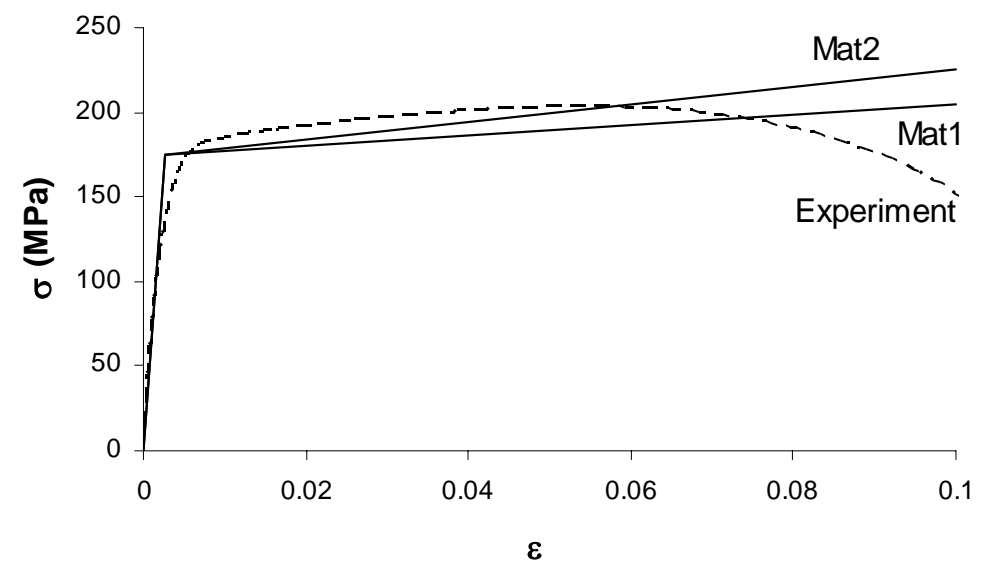

Figure A2.2 


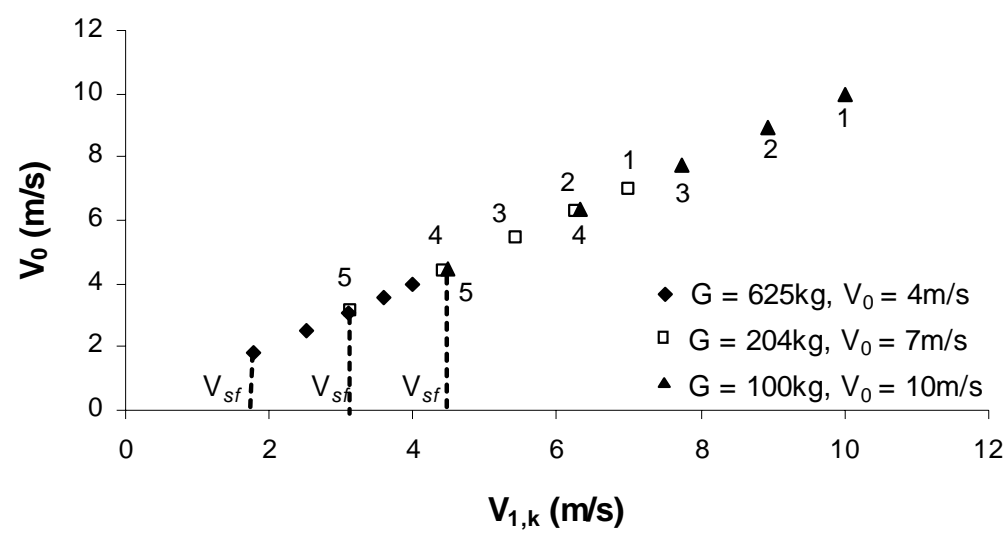

(a)

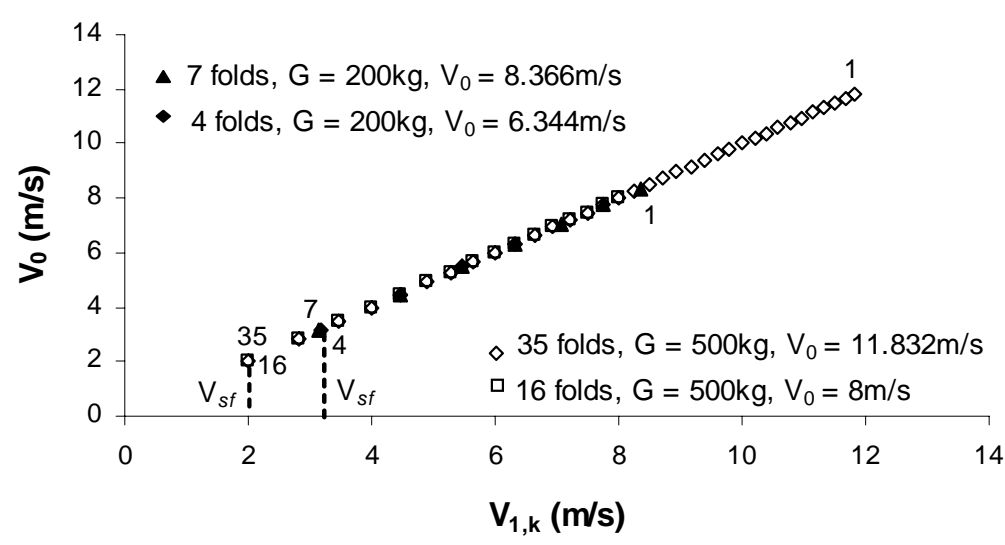

(b)

Figure A2.3 